\title{
Intense training in sport: Monitoring the effects on immune function and mood state
}

\author{
Michelle Bartlett \\ West Virginia University
}

Follow this and additional works at: https://researchrepository.wvu.edu/etd

\section{Recommended Citation}

Bartlett, Michelle, "Intense training in sport: Monitoring the effects on immune function and mood state" (2006). Graduate Theses, Dissertations, and Problem Reports. 2474.

https://researchrepository.wvu.edu/etd/2474

This Thesis is protected by copyright and/or related rights. It has been brought to you by the The Research Repository @ WVU with permission from the rights-holder(s). You are free to use this Thesis in any way that is permitted by the copyright and related rights legislation that applies to your use. For other uses you must obtain permission from the rights-holder(s) directly, unless additional rights are indicated by a Creative Commons license in the record and/ or on the work itself. This Thesis has been accepted for inclusion in WVU Graduate Theses, Dissertations, and Problem Reports collection by an authorized administrator of The Research Repository @ WVU. For more information, please contact researchrepository@mail.wvu.edu. 
Intense Training in Sport: Monitoring the Effects on Immune Function and Mood State

Michelle Bartlett, B.S.

Thesis submitted to The School of Physical Education

At West Virginia University in partial fulfillment of the requirements

for the degree of

Master of Science

In

Sport \& Exercise Psychology

Edward Etzel, Ed.D., Chair

Samuel Zizzi, Ed.D.

Ed Jacobs, Ph.D.

School of Physical Education

Morgantown, WV

2006

Keywords: Overtraining, Immune System, sIgA, swimmers, chronic stressors, mood state, training 


\section{Abstract \\ Intense Training in Sport: Monitoring the Effects on Immune Function and Mood State Michelle Bartlett}

To enhance and optimize sport performance, athletes are training at increasingly higher levels of intensity, volume and frequency. When increases in any of these areas of training are not accompanied by adequate recovery, over time, decrements in performance and physical/psychological maladies can result. Consequently, the purpose of this study was to monitor five competitive swimmers during a swimming season in two of the potential areas in which an adverse response to training may appear: the immunological parameter of mucosal immune function and the psychological parameter of mood state. Each parameter was assessed once per week over the seven weeks of the study. Immune function was assessed by salivary Immunoglobin-A concentrations analyzed by an indirect competitive immunoassay. Mood state was assessed with the Brief Assessment of Mood State (BAM; Dean et al., 1990) which is a shortened version of the Profile of Mood States. A single-case study design was used to ensure that individual differences were not masked by group means. Results of the study for the five participants show mixed results, with much variability. Data for one of the five participants supported hypothesized results where an increase in total mood disturbance and consistently low sIgA levels were demonstrated over the study. Thus, illustrated is the importance of using single-case studies to monitor the training of athletes and need to explore other test parameters for use in training studies. 
Acknowledgements

I would like to thank my chair and advisor Dr. Edward Etzel for his patience, guidance, expertise and insight throughout this entire process. The time and effort that you put into ensuring that I completed this project was unrivaled. Had you not suggested this topic in an effort to combine my interests I may not have ventured into this area in which I am truly passionate about. Furthermore, the knowledge and experienced I have gained from working with you has positively helped on my journey in learning the lessons that one can’t learn from a book. Thank you for helping me to this milestone.

I would like to thank Dr. Sam Zizzi for serving on my committee and providing me with support and feedback. The statistical and methodological expertise that you have provided throughout the study was extremely helpful. Without your suggestions on the forefront, this study would not have evolved to what it is today. Thank you.

I am also grateful to Dr. Edward Jacobs. I appreciate the time that you took out of your busy schedule to serve on my committee. Your words of encouragement and support and the interest that you have taken in this project will not be forgotten.

A special thanks to Dr. Andrew Ostrow, who invested a large portion of time into the early phases of this process. Your keen eye for detail and research knowledge has helped me greatly.

Thank you to Dr. Dan Hursh, for lending his expertise in helping me to interpret the results of this study. Because single-case interpretation is subjective, having your support behind my interpretations has given me confidence in presenting this research.

I would also like to thank Sergio Lopez and the WVU Swimming Team for allowing me use of their time and their willingness to support my study.

To the sport and exercise psychology graduate students, thank you. I am grateful to be among such intelligent and motivated individuals. I'd like to credit all of you who have shown me the ways of getting the job done and provided me with feedback.

Lastly, I would like to thank my mother whose support throughout this process cannot be expressed in words. You have helped me in ways that this study could not have been completed without you. Thank you so very much. 
Table of Contents

Page

Abstract

Introduction

Method

Participants

Research Design

7

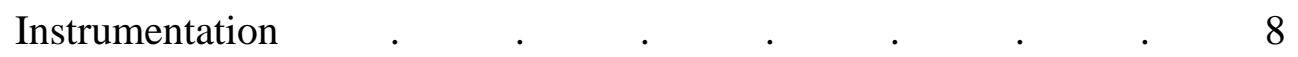

Results

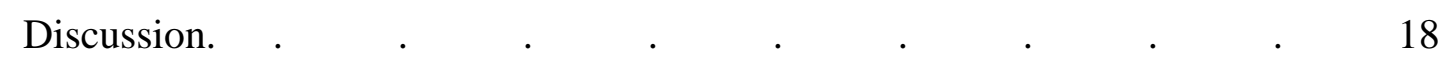

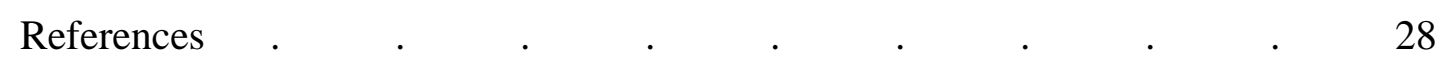

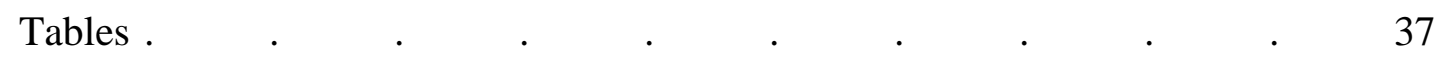

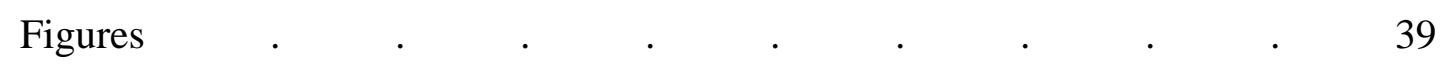

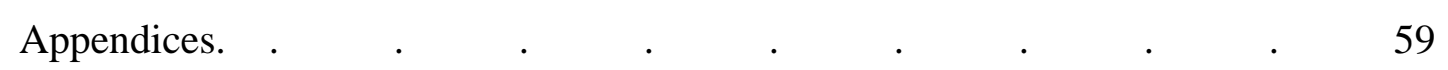

A. Brief Assessment of Mood State Questionnaire. . $\quad$. $\quad$ 59

B. Training Load/ Illness Assessment . $\quad$. 60

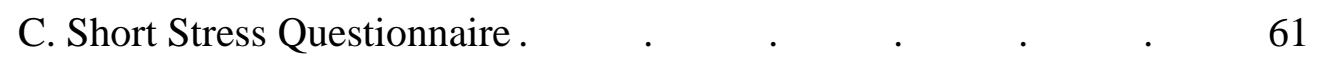

D. Approval from the Institutional Review Board for the
Protection of Human Rights

E. Consent and Information Forms $\quad$. $\quad . \quad$. $\quad$ • 65

F. Demographics Questionnaire. $\quad \quad \quad \quad \quad \quad \quad \quad \quad \quad 69$

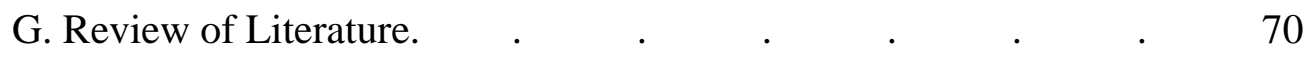




\section{List of Tables}

Page

Table 1: Means and Standard Deviations of Mood State, Life Stress and sIgA

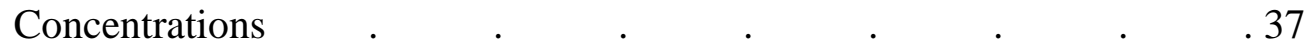

Table 2: Bivariate Correlations of Test Variables for Participants _ 


\section{List of Figures}

Page

Figure 1: Individual training load per week for each participant, measured in yards over seven weeks. $\quad . \quad$. $\quad . \quad$. $\quad$.

Figure 2: Mood, as measured by the BAM, and life stress, as measured by the short stress questionnaire, over 7 weeks for P1.

Figure 3: Salivary IgA concentration in ug/mL, measured once per week over seven weeks for P1. $\quad . \quad$. $\quad$. $\quad$.

Figure 4: Mood, as measure by the BAM, and life stress, as measured by the short stress questionnaire, over three consecutive days during Week 1 for P1. $\quad$. $\quad$. $\quad$. $\quad$.

Figure 5: Mood, as measured by the BAM, and life stress, as measured by the short stress questionnaire, over 7 weeks for P2. . .

Figure 6: Salivary IgA concentration in ug/mL, measured once per week over seven weeks for P2. $\quad . \quad \quad$. $\quad$. $\quad$.

Figure 7: Mood, as measure by the BAM, and life stress, as measured by the short stress questionnaire, over three consecutive days during Week 1 for P2. $\quad . \quad$. $\quad . \quad$.

Figure 8: Salivary IgA concentration in ug/mL, over three consecutive days during Week 1 for P2.. . . . . .

Figure 9: Mood, as measured by the BAM, and life stress, as measured by the short stress questionnaire, over 7 weeks for P3. . 
Figure 10: Salivary IgA concentration in ug/mL, measured once per week over seven weeks for P3. $\quad$. $\quad$. $\quad$. $\quad$.

Figure 11: Mood, as measure by the BAM, and life stress, as measured by the short stress questionnaire, over three consecutive days during Week 1 for P3. $\quad$. $\quad$. $\quad$. $\quad$.

Figure 12: Salivary IgA concentration in ug/mL, over three consecutive days during Week 1 for P3. . $\quad . \quad$. $\quad . \quad$. $\quad$. $\quad$. 50

Figure 13: Mood, as measured by the BAM, and life stress, as measured by the short stress questionnaire, over 7 weeks for P4. . $\quad$. . 51

Figure 14: Salivary IgA concentration in ug/mL, measured once per week

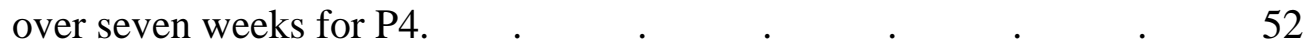

Figure 15: Mood, as measure by the BAM, and life stress, as measured by the short stress questionnaire, over three consecutive days during Week 1 for P4. $\quad . \quad$. $\quad . \quad . \quad . \quad$.

Figure 16: Salivary IgA concentration in ug/mL, over three consecutive days

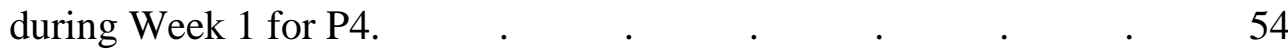

Figure 17: Mood, as measured by the BAM, and life stress, as measured by the short stress questionnaire, over 7 weeks for P5. . $\quad . \quad$. 55

Figure 18: Salivary IgA concentration in ug/mL, measured once per week

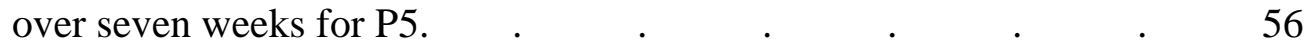

Figure 19: Mood, as measure by the BAM, and life stress, as measured by the short stress questionnaire, over three consecutive days during Week 1 for P5. 
Figure 20: Salivary IgA concentration in ug/mL, over three consecutive days

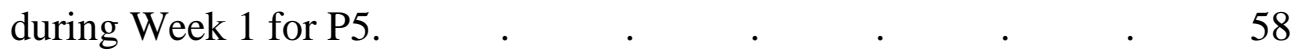


Intense Training in Sport: Monitoring the Effects on Immune Function and Mood State

Olympic motto 'Citius, altius, fortius' ('faster, higher, stronger') ultimately defines the goal of training. Coaches and athletes are continuously trying to find ways to sustain improvement. To enhance and optimize sport performance, athletes are training at increasingly higher levels of intensity, volume and frequency. However, there is no definitive training theory that describes the type, quantity or pattern of training that guarantees a given level of athletic performance (Lehmann, Foster, \& Keul, 1993; Pyne et al., 2000). When increases in any of these areas of training are not accompanied by adequate recovery, decrements in performance and psychological and/or physical maladies can result, as well as permanent exit from sport (Lehmann et al., 1993).

The term 'overtraining' has be used to describe the syndrome that may occur when more training produces lower performance instead of enhanced performance, and many other negative and/or counterproductive psychological, physiological, immunological and biological symptoms (Fry, Morton \& Keast, 1991). Main symptoms may include intense fatigue, underperformance, muscle soreness, reduced appetite, mood disturbances, and disturbed sleep patterns (Budgett et al., 2000; Gleeson, 1998; 2002; Hassmen, 1998; MacKinnon, 2000; McKenzie, 1999). Practically, athletes and coaches need to be able to identify overtraining and under-recovery symptoms as early as possible in order to alter training before performance deteriorates.

The number of athletes affected and the range of detrimental effects that may impact an athlete's participation illustrates the importance of studying training and its 
effects. For instance, the importance of optimal training is evident in the very small difference between winning and losing at elite levels. A competitively meaningful performance among athletes may only be on the order of 1\% (Lehmann et al., 1993).

MacKinnon (2000) explained that the prevalence of overtraining varies by sport and is deemed to be highest in endurance sports requiring high volume intense training such as swimming, triathlon, road cycling, rowing and running. Some studies focus solely on overtraining in endurance sports (i.e., Gabriel, Urhausen, Valet, Heidelbach, \& Kindermann, 1998; Lehmann et al., 1993; Petibois, Cazorla, Poortmans, \& Deleris, 2003; Robson, 2003). However, overtraining has been identified in most sports - both endurance and power sports.

As long as athletes seek to improve their own or record performances, overtraining, both as a process and product will endure. Taking into account the prevalence and potential impact of overtraining, it is curious that there has yet to be a definitive test established that can clearly identify when an athlete is responding negatively to training. The underlying mechanism for performance decrements is not fully known (Armstrong \& VanHeest, 2002; Fry et al., 1991; Hawley \& Schoene, 2003; Urhausen \& Kindermann, 2002). The onset and progression of overtraining are not easily predictable: methods for monitoring are more practical in the research laboratory than in the athletic arena (Lehmann et al., 1993). Clearly, there is a need for relevant tools for the early diagnosis of overtraining (Urhausen \& Kindermann, 2002).

\section{Biological Responses}

There have been many proposed markers for the early identification of overtraining. It has been suggested that altered immune reactions might provide an early 
warning sign that an athlete is overtraining (McGrew, 1995). A common symptom of overtraining is immunosupression, characterized by slower wound healing (Newsholme, 1994) and an increased susceptibility to infection, namely upper respiratory tract infections (URTI) (Fitzgerald, 1991; MacKinnon, 2000; McKenzie, 1999; Neiman, 1994; Shephard \& Shek, 1998; Smith \& Norris, 2002). For example, in a study of 24 competitive swimmers by MacKinnon and Hooper (1996), training was intensified over 4 weeks resulting in $33 \%$ of the swimmers showing symptoms of short-term overtraining. Of those 24 swimmers, 42\% were diagnosed with URTI's. A study of 25 elite level speed skaters by Foster (1998) attempted to define the percentages of illness that could be correlated to each index of training: load, monotony and the product of the two factors combined - strain. An analysis of training journals ranging from six months to three years, it was found that $84 \%$ of illnesses followed a spike in training load above the individual training threshold.

It has been also argued that levels of total secretory IgA measured in saliva are representative of immunological activity in the immune system as a whole (Evans, Hucklebridge, \& Clow, 2000). Host defense at mucosal surfaces from invading pathogens is mostly mediated by the family of secretory immunoglobins (Ig) (MacKinnon, 1996). The principal antibody, or immunoglobin, found in external secretions, such as mucous, tears, and saliva, is Immunoglobin A, or IgA (Gleeson, 2002; Nieman, 1994). To date, salivary IgA concentration is the only immune parameter to be directly associated with the appearance of URTI’s (MacKinnon, 2000) and is used a marker for athletes at risk of developing an URTI (Gleeson, 2002; Shephard \& Shek, 1998). Lower levels of salivary IgA have been documented in over 
trained athletes and decreases in salivary IgA concentrations can be observed during intensified training in elite swimmers (MacKinnon, 1996). For example, in a study by MacKinnon and Hooper (1994) a significantly lower concentration of salivary IgA was reported for a group of elite swimmers throughout a six-month season who were clinically diagnosed as suffering from overtraining, compared to teammates that were considered well-trained. In another study, Gleeson, McDonald, et al. (1995) found a significant decrease in both pre- and post-exercise sIgA levels over a seven-month training period with elite swimmers compared to age-matched non-athletes. Data from this study also indicated that the elite swimmers with low sIgA levels at the start of their season were associated with a higher risk of RTIs during the season (Gleeson, Hall, et al., 1999).

Psychological Responses

Some researchers have argued that psychological testing is very effective in detecting overtraining at an early stage (Kentta \& Hassmen, 2002; O’Connor, 1998; Shephard \& Shek, 1994). Athletes who respond adversely to training tend to show a trend in the order of the indices affected with the first noticeable change suggested to be within psychological factors, followed by medical condition, and lastly performance (McKenzie, 1999). Psychological and emotional symptoms indicative of overtraining include unstable and/or depressed moods, lowered tolerance of frustration and stress, feelings of helplessness and worthlessness, a fear of competition, anxiety and apathy, and sleep disturbances (Fry et al., 1991; Henschen, 2000).

Shephard and Shek (1994), and Davis and Colbert (1997) and reviews of the literature on markers of overtraining indicate that psychological assessments are most 
reliable across athletes. This finding coupled with the inconsistency of physiological markers, as well as the early onset of psychological symptoms, suggests psychological testing as a more effective way to assess overtraining in athletes than physiological measures.

Morgan, Brown, Raglin, O’Connor and Ellickson (1987) and Morgan, Costill, Flynn, Raglin, and O’Connor (1988) demonstrated that psychological testing is a highly sensitive overtraining early warning marker in their studies of competitive swimmers. Overall, they found that there is a dose-response relationship where increased training is associated with increased mood disturbance. The earliest psychological changes are an increase in fatigue and a decrease in vigor. Mood profile monitoring may successfully predict the physiological and performance changes associated with overtraining. Psychoneuroimmunological Responses

The data of MacKinnon and Hooper (1994) show a significant negative correlation $(p<.02)$ between sIgA concentrations and indicators of mood state disturbance. They also suggest that mucosal immunity may be compromised by daily exercise of at least moderate intensity when undertaken by competitive athletes. Thus, an athlete may show impaired immunity as a result of training but may not actually be over trained. However, the product of impaired immunity and a disturbed mood state may be a noteworthy marker of overtraining. By combining these potential markers of overtraining and comparing the relationship between the two, an early warning of counterproductive training effects can be detected before performance decrements have a serious impact on an athlete's function. 
This interdisciplinary approach lays within the realm of psychoneuroimmunology- the study of the behavioral- neural- endocrine- immune system interactions, or the relationship between psychological variables, the nervous system, and the immune system (Ader \& Cohen, 1995).

\section{Limitations in Previous Research and Application}

To advance toward prevention and early recognition of negative psychological and immunological effects of intense training, Hassmen (1998) called for better understanding in several areas: the lack of accepted tests and standard criteria to unerringly place athletes on the training-response continuum, lack of a reliable and easily managed method to monitor responses to training, the need to define markers of recovery, and the integration of these factors.

A preventive approach in marking overtraining is more time and cost-effective than treatment post-diagnosis. With reliable indicators of adverse psychological and immunological effects, such as what this study sought to find, athletes may avoid needing long time periods away from training to recover, and save on medical costs for treatment and rehabilitation. Evaluating athletes individually, monitoring them regularly and comparing data longitudinally to their own baseline values could be keys to the early identification of adverse training (Kellman, 2003). Individual monitoring can allow coaches and athletes to then modify training programs to minimize counterproductive immunological and psychological effects at critical time periods while still moving forward with training.

Single-case studies on training effects are rare. Most previous work has been conducted with group comparisons. Grouping data for statistical analysis often masks 
individual variability. The single-case analysis in this study allows for the isolation of intra-subject variability, and thus yields the ability to state more clearly the conditions under which the hypotheses does and does not provide a useful model.

The primary purpose of the study was to investigate the effects of an accumulating training load on five individual athlete's mood state and immune function over seven weeks during the early half of an intercollegiate swimming season and to see if there is a relationship between the two dependent variables. It was hypothesized that as the season progresses and training load accumulates, total sIgA concentration will decrease indicating decreased immune function and BAM scores will increase showing an increased mood disturbance. To minimize incorrectly attributing changes in the dependent variables to training effects, a secondary purpose of the study was to explore if stress from sources outside of athletics and/or illnesses coincided with changes in these variables.

\section{Method}

\section{Participants}

The participants were five $(n=5)$ female National Collegiate Athletic Association (NCAA) Division 1 middle-distance to distance swimmers (approx. mean age $=20$ years) at a large mid-Atlantic university. With IRB approval, these individuals were randomly selected by their coach as potential participants meeting basic requirement for participation and subsequently agreed to participate. Participants were all actively training with the team at the time of the study. 


\section{Research Design}

This study employed a descriptive, uncontrolled single-case study with a 'B'design (Barlow \& Hersen, 1984). This design allowed for the study of the effects of the independent variables. It was a "naturalistic study" (Barlow \& Hersen, 1984) because the researcher did not manipulate the independent variables, but studied the athletes over a large part of a competitive collegiate training season. A control group was not employed in this study. This was the case because a suitable control for these highly trained competitive athletes was unavailable.

\section{Instrumentation}

Mood state. Mood state is the psychological variable assessed in this study. It was assessed using the "Brief Assessment of Mood State” (BAM; Dean et al., 1990) (see Appendix A). The BAM is a shortened version of the "Profile of Mood States" (POMS; McNair et al., 1971). The POMS is a 65-item self-report questionnaire, which yields a global measure of mood as well as the stable mood states of tension, anger, vigor, fatigue, and confusion.

The BAM contains six questions that inquire about anxiety, sadness, depression, confusion, energy, fatigue and anger to measure mood. Participants are asked to respond in accordance with "how I feel right now" instructions on a five-point ordinal scale. The total BAM “Total Mood Disturbance” score (or TMD score) is calculated by summing the six BAM items while weighing the "energetic" item negatively. Therefore, a higher BAM score is indicative of a more negative mood state.

Sufficient reliability and validity coefficients have been reported for this test. In a large validity study with collegians ( $N=621)$, acceptable correlations between BAM 
items and POMS counterpart scales ( $r=.66-.87)$ were reported. The Cronbach's coefficient alpha for the POMS TMD was .85 and .75 for the BAM TMD. The Pearson product-moment correlations for the POMS TMD and the BAM TMD is high, $r=.88$, $p=<.001$, all indicating high criterion and concurrent validity for the BAM (Dean et al., 1990). It takes approximately thirty seconds to complete the BAM.

Immune function. Levels of total secretory IgA measured in saliva are representative of immunological activity in the immune system as a whole (Evans et al., 2000). Therefore, measuring salivary IgA concentrations assessed immune function. Saliva (“passive drool”) was collected in a $2.0 \mathrm{ml}$ vial, sealed tightly and stored in a non-cycling freezer at ${ }^{-} 60-{ }^{-} 80^{\circ} \mathrm{C}$. At a cost of $\$ 18.95$ per assessment, these measurements were determined by an indirect competitive immunoassay (Salimetrics, State College, PA). For salivary IgA, intra-assay variance is $5.7 \%$ and the sensitivity of the assay is $2.5 \mu \mathrm{g} / \mathrm{mL}$ (Salimetrics, State College, PA). Reference ranges for normal adults fall between 10 and 105 mg/L (Gleeson, Hall, et al., 1999).

Training load and illness (See Appendix B). A brief questionnaire was given with two open-ended questions: 1) “How many meters have you swum in the past week?” and 2) "Have you experienced any respiratory infections (defined as symptoms of fever, pharyngitis, tonsillitis, rhinitis and swollen glands consistent with either overt bacterial infection or viral illness) (Gleeson, Hall, et al., 1999) in the past week? If yes, please briefly specify.”

Research has shown that there is a correlation between training load and infection (Neiman, 2000). Low salivary IgA levels are correlated with increased risk of developing infections (Gleeson, Hall, et al., 1999). At moderate training loads, salivary 
IgA levels show no change (McDowell et al., 1992). Some studies demonstrate that increasing training loads during a competitive season act as a chronic stressor on immune function (Gleeson, McDonald, et al., 1999; Gleeson, Hall, et al., 1999; MacKinnon \& Hooper, 1994; Nieman, 2000; Tharp \& Barnes, 1990). Training load, and not training intensity, was assessed as Gleeson, McDonald et al. (1999) noted that training intensity and volume were two relatively fixed patterns over a training period and at any given time there was little variation in training volume/physical intensity combinations at highly competitive levels.

Life stress. To assess life events that could be a source of stress beyond the physical and psychological stress of intense training, a short stress questionnaire created by the author was administered. (See Appendix C). The scale incorporated seven items with a nine-point Likert scale. The participants were to answer each question if they had experienced stress in the areas of: 1) academics, 2) social/personal, 3) family, 4) financial, 5) self-image, 6) health, and 7) athletics “within the past two months”. The scale ranged from 0 to 8 with “ 0 ” indicating no stress in that area to " 8 ” indicating extreme stress. This data was used post hoc to compare with variations in measurements of target dependent variables.

The items on this questionnaire were derived from the "College Stress Questionnaire” (Otani, 1985) and the “WVU Quick Stress Questionnaire,” which was modified from Otani (1985). The WVU Quick Stress Questionnaire (QSQ) is a 25 item, nine-point Likert-type subjective paper-and-pencil questionnaire. It was designed to assess both sources of stress and stress symptom manifestations. The questionnaire is organized into halves. The first eight items on the scale reflect sources of stress: 1 ) 
academic/work, 2) social/personal relationships, 3) family concerns, 4) financial concerns, 5) self-image, 6) health concerns, 7) sexual concerns, 8) day-to-day hassles, and 9) “other”. The second 12 items reflect the stress symptoms that may be present: 10) depression, 11) anger, 12) fear, 13) muscle tension/aches, 14) indigestion, 15) tics/tremors, 16) sleep, 17) eating, 18) drinking, 19) forgetfulness, 20) hypertension, 21) dermatological, 22) avoidance behavior, 23), 24), 25) overall stress levels. The items chosen for this short stress questionnaire reflect those items on the "Perceived Impact of Potential Adjustment Factors” (PIPAF; Downey, 2005), which is a questionnaire designed to assess the perceived impact of potential stressors on the adjustment to college.

Procedure

Prior to collecting data, approval was obtained from the team coach and the Institutional Review Board for the Protection of Human Subjects (see Appendix D). An informed consent letter (see Appendix E) was distributed before data collection outlining the nature of the study and the participant's rights as a research participant. To protect the confidentiality of participants, a randomly assigned, confidential identification code number was assigned to each participant in the study. The informed consent letter also explained that by completing and returning the informed consent form and questionnaires, the individual was agreeing to participate in the study.

To examine day-to-day stability of the measures, the first week of the study incorporated assessments on three consecutive days. During first of the assessment meetings, the participants first completed a demographic questionnaire (see Appendix G). To maintain anonymity, each participant was assigned an identification number 
from a master list, which was used throughout the study. On all of the assessment meetings, including the six weekly assessments thereafter, to minimize the effects of circadian rhythms, data was collected at the same time of day. Testing was done before the start of a training session as this allows a rest period to exclude potential residual effects of a previous training session and ensure that any marked differences are reflecting cumulative effects. Salivary IgA samples were collected first. The vials were sealed and marked with identification number. Participants then completed the paperand-pencil BAM. Items on the BAM were answered with respect to how one has been feeling "the last week including today." Lastly, the short stress questionnaire and the two open-ended questions concerning training volume and incidence of illness were answered. Saliva samples were stored in a non-cycling freezer $\left({ }^{-} 60-{ }^{-} 80^{\circ} \mathrm{C}\right)$ for the length of the study. Upon cessation of the study, samples were shipped to the lab for analysis. 
Results

Data Analyses

The Microsoft Excel statistical package (Microsoft, Inc., 2004) was used to provide a visual depiction of data. Four graphs were created for each participant to illustrate data: mood and life stress over 7 weeks; sIgA concentrations over 7 weeks; stability of mood and life stress over 3 consecutive days; and stability of sIgA over 3 consecutive days. Training load for each participant was combined into one graph. Estimated, individual weekly training loads for each participant are found in Figure 1. Participants’ data are provided in Figures 2-20.

Changes in mood state, immune function, and life stress were analyzed using a combination of visual inspection and descriptive statistics. Because the design of the study incorporated only one phase, only within-phase analyses, such as amplitude (intensity), variability and trend were used in the visual analyses. Amplitude is the intensity of the scores on the measurements. "Variability" is the fluctuation of each participant's sIgA and mood state over time and "trend" is the tendency for the variables to decrease or increase systematically or consistently over time (Kennedy, 2005)

Using SPSS (SPSS, Inc., 2001), descriptive statistics and Pearson product moment correlations were conducted for mood state, sIgA, and life stress for each participant over the study to evaluate if any of the variables change similarly over time. Participant 1

Participant 1 (P1), had reportedly been swimming competitively for 12 years, 1.5 years on the current team, and was currently at her highest competitive level. She 
reported an estimated average of two URTI's per year; she did not report experiencing any URTI's over the course of the study. In Figure 2, a flat slope indicates that after an increase in BAM scores between week 1 and 2, the mood scores of P1 remained stable over six weeks. Average TMD throughout the study was the highest among all participants $(M=10, S D=3.37)$ (see Table 1$)$. Figure 3 shows sIgA values with a great deal of variability, with no trend patterns over the study. The split-middle method of trend estimation revealed a somewhat positive trend of medium magnitude for sIgA over the seven weeks, inconsistent with hypothesized behavior. Conversely, a decreasing trend was seen over weeks 5, 6, and 7, in support of the hypothesized decrease of sIgA as training load accumulated. A comparison of BAM scores and sIgA concentration with life stress scores, showed stability over time, with only a slight decreasing trend of low magnitude over time. The stability of mood and life stress over the seven weeks is shown in Figure 4. Aside from a slight increase in life stress and a negative trend in sIgA during the last week, there was no observable relationship evident between life stress and sIgA. Due to an insufficient amount of sIgA sample collected for the first test point, and the recommendation of least three initial data points (Barlow \& Hersen, 1984), sIgA stability over three consecutive days for P1 could not be determined.

Participant 2

Participant 2 (P2) had reportedly been swimming competitively for 15 years, 3 on her current team. P2 estimated experiencing an average of 4.5 URTI’s per year, 3 during the study. Figure 5 shows that P2's mood remained fairly stable. A decrease in TMD between week 1 and 2 and an increase in TMD between week 6 and 7 is evident. 
However, overall for mood there is a flat trend. In Figure 6, a flat trend is also shown for sIgA over the 7 weeks, although there is some variability around that trend line, especially at weeks 5 and 6 . At week 6, there appeared to be a taper (i.e., intentional training load reduction) of training (see Figure 1). Subsequent to taper, life stress and TMD both decreased, which could be expected. However, sIgA decreased during tapering, which was unexpected in light of the hypothesized dose-response relationship. Also during this time, P2 reported sickness during weeks 4, 5, and 7. This was not the case when sIgA was lowest during week 6, which is inconsistent with previous research supporting lowered sIgA and incidence of URTI. Life stress scores varied similarly with mood over the seven weeks. sIgA and life stress also followed vary similarly over the study, which however was not expected as they were expected to demonstrate an inverse relationship.

A flat trend in mood was observed day-to-day during week 1 (see Figure 7), consistent with the observed flat trend and limited variability seen for mood over the study. At that time, a slight decreasing trend in sIga level was seen (see Figure 8).There was considerable variability among a small number of data points, indicating that making accurate inferences from this data is difficult.

\section{Participant 3}

P3 had reportedly been swimming competitively for 11 years, one being on the current team. P3 reported having swum at the elite/ Olympic level. P3 reported an estimated average of one URTI per year, two during the study. Allergies were also reported at week 7. Figure 9 shows mood and life stress for P3 varied similarly following the same low-magnitude negative trend. There is little variability around the 
trend aside from a marked increase in both TMD and life stress around week 4 . In the weeks following this increase, the participant reported sickness for the subsequent weeks in the study. Figure 10 shows that during week 6, one week after the participant first reported sickness, sIgA also decreased, corresponding with a taper in training load at that time (see Figure 1). SIgA increased dramatically at week 7 to almost four times the average sIgA level for this participant. For P3, three-day stability measures for life stress (see Figure 11) and sIgA (see Figure 12) show large variability, with mood remaining stable (see Figure11).

\section{Participant 4}

P4 reported swimming competitively for six years, 1.5 of those being on the current team. P4 estimated having an average of 5.5 URTI's per year, and reported a sickness at every test point during the study. It is unclear whether or not this was the same sickness throughout the study. P4 missed the testing for week 2 and only training load could be assessed retrospectively. Figure 13 shows an increasing trend of medium magnitude for TMD, although mood varied moderately through the study. This supports the hypothesis that as the season progresses and training load accumulates, mood will be adversely affected. Figure 14 shows the large variability amongst sIgA values over the study with a very low-magnitude increasing trend. Aside from the sIgA value from week 5, which is marked by a drastic increase in intensity, sIgA showed a consistent flat trend, at a low sIgA concentration. During weeks 6 and 7, sIgA remained at low levels, also supporting the hypothesis that as the season progresses and training load accumulates, sIgA will be adversely affected. It also should be noted that P4 reported sickness at every of the test points, supporting previous research that lower sIgA levels 
will yield a decreased immune function and greater incidence of sickness. Concerning life stress as a potential source of variability, as shown in Figure 13, life stress followed a mid-magnitude decreasing trend, with moderate variability. As the other two variables followed the hypothesized effect due to an accumulating training load over the study, life stress actually decreased for this participant, ruling out the possibility that increasing life stress, as opposed to training load, was relational to the dependent variables.

Concerning stability over three consecutive days for the variables of interest, Figure 15 shows mood to be fairly stable and life stress showing a medium-magnitude decreasing trend. Figure 16 shows sIgA over three consecutive days to exhibit large variability, as it had through the course of the study.

\section{Participant 5}

P5 reports swimming competitively for 12 years, having competed at the elite/ Olympic level. P5 had been on the current team for less than one year. P5 reported an estimated average of five URTI's per year and did not report any through the course of the study. Figure 17 shows mood and life stress varying similarly over time with only slight variability. Mood and life stress for P5 were highly correlated $(r=.889, p<.01)$ (see Table 2). Using the split-middle technique, which uses the median of the data points to estimate trend, a slightly negative trend of low-magnitude is evident. However, in using a regression line, which uses the mean of the data points to estimate trend, a stronger high-amplitude negative trend is seen for life-stress and a midmagnitude negative trend is seen with mood. This does not support the hypothesized relationship of mood and training load accumulation over the season. Figure 18 
demonstrates sIgA as following a cyclical pattern with almost no variability at all. Overall, a large-magnitude increasing trend is present. At the taper in training load during week 6 (see Figure 1), sIgA increased (see Figure 18), which would have been expected in line with the purported dose-response relationship. sIgA then began to decrease thereafter.

Concerning the stability of the measures over 3 consecutive days, the demonstration of little variability for mood, life stress (see Figure 19) and sIgA (see Figure 20) supports the behavior of the variables over the course of the study.

\section{Discussion}

The primary purpose of this research was to monitor the individual sIgA, mood and training loads of intensely training swimmers within the earlier half of the participant's season and to discover if any relationships were exhibited among the variables. Overall, there was much inter- and intra-variability evident in the data. Inconsistent support was obtained for the hypothesized relationships among the variables. Of the five participants, only the data of $\mathrm{P} 4$ exhibits trends consistent with the hypothesized changes - an increase in TMD and lowered sIgA levels, which were not correlated with life stress, as training load accumulated over the study.

\section{Mood State}

Although it can be difficult to predict or diagnose adverse training effects from mood state self-report questionnaires, it was expected that the participants would demonstrate an increase in TMD in response to an accumulating training load over the study. Only P4 demonstrated a consistently increasing TMD in a dose-response manner. Also, P1 demonstrated a stable higher TMD than the rest of the participants throughout 
the study. As this study failed to do, it would be interesting to see P1's baseline mood state and at the very beginning of the swimming season, where an increasing trend may have existed.

Of the five participants, only one (P5) showed a significant correlation among mood and life stress scores $(r=.889, p<.01)$. However, this may have been due to the small number of data points analyzed for each participant. If a correlation did indeed exist among the variables for any participant, having more data points would increase the possibility of a significant finding. Hooper et al. (1997) showed that POMS (of which the BAM is a shortened version) scores were more closely related to training intensity, as opposed to training volume, the variable measured in this study. Thus, assessing training intensity as an independent variable could possibly yield a significant relationship with mood state. It should also be noted that as while the dose-response relationship for TMD and training volume is consistently supported in the literature (see Appendix G), none of those studies utilized the questionnaire that was used in this study. The majority of studies showing a relationship between training volume and mood state used the 65-item POMS questionnaire (McNair, et al., 1991). It is possible that the hypothesized increase in TMD may have been evident in this study if a more comprehensive instrument was used.

\section{Immune Function}

It was hypothesized that sIgA levels would be generally decreasing or lower over time. This was not consistently observed. P1, P4, and P5 demonstrated a strong decreasing trend during the second half of the study, but P1 and P5 did not exhibit a consistently decreasing trend through the entire duration of the study,. The potential for 
showing a strong decreasing trend in sIgA levels may have been diminished because many of the participants demonstrated occasional high sIgA concentrations. P2, P3, and P4 all demonstrated this finding where an URTI was reported followed by an increase in sIgA concentration the next week. This finding is similar to a 2002 study that used daily sIgA sampling for swimmers over 30 days (Gleeson et al, 2002). However, it is important to note that lower sIgA often precedes an infection by 48 hours (Mackinnon et al, 1993). As also shown by Foster (1998), 84\% of illnesses followed a spike in training load above individual training threshold. These phenomena were unable to be detected in this investigation because sampling was only done once per week. Infrequent sampling may have also masked the relationship between training and preexercise sIgA levels that was demonstrated in a study by Novas, Rowbottom, and Jenkins (2003) where sIgA concentration was directly associated with the amount of training undertaken on the previous day $(p<.05)$.

As evident in this study, it is possible that elite swimmers are prone to great biological variation. Francis, Gleeson and Pyne, et al. (2005) studied the variation of sIgA among swimmers and sedentary populations. Overall, elite swimmers compared with active and sedentary individuals had higher concentrations of sIgA and greater variability in sIgA concentrations as individuals and as a group. Their findings are consistent with the findings of the current study in which most participants showed large variability, ranges, and fluctuation. Normal ranges for athletes have not been established for tests of immunocompetency (Bagger, Petersen, \& Pedersen, 2003; Pyne \& Gleeson, 1998). Therefore, caution should be taken when attempting to compare the participants of the current study with each other or to a general population. 
Other studies have also not found significant trends for sIgA levels over training periods such as with elite swimmers (Gleeson et al., 2000; Mackinnon \& Hooper, 1994) collegiate swimmers (Tharp \& Barnes, 1990), “endurance-trained” cyclists (Halson, Lancaster, Jeukendrup, \& Gleeson, 2003), collegiate soccer players (Putlur et al., 2004), male basketball players (Tharp, 1991), It should be cautiously noted that these studies used group designs, as opposed to single-subject, so individual changes may have been masked.

Psychoneuroimmunological Implications

Psychoneuroimmunology emphasizes the bi-directionality of communication between the immune, endocrine and nervous system. Thus, all three systems have the ability to influence the function of one another (Ader \& Cohen, 1995)

Cortisol, the primary hormone that is released in the body in response to stress, is a critical variable that was not measured in this study. The current study did not uncover any strong relationships among immune function, mood and training. Training volume represented the source of physiological stress and life stress represented the source of psychological stress for the participants and discovering their cumulative effect on mood and immune function over time was an objective. Instead of indirectly measuring stress, such as interpreting an increasing training volume as increasing stress, measuring cortisol levels directly would have been a direct method in which stress for the participants could have been measured. Instead of assuming the interpretation of the stressors by each participant in a dose-response manner, cortisol levels could have provided an objective measurement of the stress that each was experiencing. For 
instance, the testosterone- cortisol ratio has been considered as a meaningful measurement of stress in females (Lac \& Berthon, 2000) and a 30\% decrease in this ratio is recommended as a useful parameter in the early diagnosis of adverse training effects (Lehmann et al., 1993).

While stress may activate an immune response, the mounting of an immune response leads to the physiological equivalence of a stress response. The same mechanisms activate both an immune response and the response to stressors along the HPA axis (Maier, Watkins, \& Fleshner, 1994). This implies that the dependent variables may not have been independent of one another, but in fact influencing or affecting each other. However, this is not evident here because there were no strong correlations among mood state and sIgA in the data sets.

\section{Limitations}

Because this study did not strongly support the hypothesized changes in the dependent variables, it can be postulated that per participant, too few assessments were taken over the study to show significant change It is possible that a two-month assessment period is not sufficient for the assumed changes in the variables to manifest themselves. In studies by Gleeson, et al. (1995;1999) swimmers showed a progressive decline in sIgA levels over seven months, supporting the possibility that seven weeks is not sufficient. Throughout the study, variables of interest were assessed once a week. Also, in other studies of this nature the variables were assessed at shorter intervals, including daily log books used to record training and illness (Francis et al., 2005; Mackinnon \& Hooper, 1994; Novas et al., 2003; Tioller et al., 2005) daily psychological assessment (Halson et al., 2003) and thrice-weekly assessments of 
biological parameters (e.g., Francis et al, 2005). It is possible that important, subtle changes in the dependent variables were missed in the once weekly assessments.

At the onset of the study, measurements of the dependent variables were taken on three consecutive days. Because participants had already been practicing for approximately 1.5 months at the time, these measurements could not be considered as a useful baseline. In any single-case study, a solid baseline plays an integral part in establishing internal validity. The lack of an established baseline for each participant made traditional pre-post comparisons unachievable. The importance of baselines for each individual athlete is highlighted in Fry et al. (1991) and Hartmann and Mester (2000). Taking into account that even during the "off season”, highly trained athletes are never "not trained", they suggested that baseline measurements must be taken for individual athletes in a normally trained state as their own control. Results are not applicable in relating to population norms because changes indicative of the onset of the negative effects of training may be too sensitive to be noted. Athletes may be in the stages of gross overtraining before clinically significant changes are noticed.

External validity is established in single-subject studies via replication among closely matched participants with the same problem/issue. Successful replication establishes generalizability (Gay \& Airasian, 2003). This study aimed to establish external validity with simultaneous replication, by using five participants at the same time and location. There were few similarities among the data of the five participants to infer generalizability of the study design for use in monitoring training effects. Whether this can be attributed to inter-individual differences in recovery potential, exercise capacity, non-training stress, and stress tolerance among the athletes under identical 
training conditions (Kellman, 2003) is yet to be determined. Bagger et al. (2003) suggested that without control data, separating training effects from natural biological variation is not possible. Therefore, this limitation can be due to methodological flaws or participant selection because they were in fact not closely matched as was assumed for this experimentally accessible population of participants. Also illustrated here is the disadvantage of this model is the inability to control for confounding variables and the history effects outside of the monitored parameters among participants.

Interestingly, the head coach of the participants used in this study was named as the Conference Coach of the Year. This recognition gives support to the possible explanation that these swimmers were in fact training properly - incorporating a wellrounded training/recovery regimen. Thus, it was possibly less likely for the swimmers to show the adverse immune and psychological effects of improper training.

\section{Future Suggestions}

Fry et al. (1991) call for the administration of a battery of tests for training athletes covering several parameters associated with homeostatic imbalances taken as a baseline measure - meaning more than only sIgA levels and mood state. In example, monitoring cortisol levels could be useful as a direct marker of stress levels in participants as opposed to using training load or intensity as an indirect marker. Evaluating athlete's training load and intensity individually, monitoring them daily, as opposed to weekly, and comparing data longitudinally could be a key in marking the negative and positive effects of training (Kellman, 2003). Also, just as there are individual variations in responses to training, there are also differences in recovery and perceptions of recovery. Subjective instruments could be utilized to assess if an athlete is recovering from training, regardless of a noticeable effect on the dependent 
variables, such as those monitored in this study. Because one’s perceptions influence one’s reality, simply asking the athletes about their views about their training and recovery could yield valuable information on training effects. Using more qualitative information, such as daily log books or training diaries, could generate more conclusive information pertaining to effects on dependent variables. In particular, Smith (2003) suggests a more specific assessment of reported URTIs, as respiratory symptoms taken alone are non-specific and quite possibly misleading. It would seem to be more appropriate to medically monitor symptoms to determine whether they are pathogenrelated or a result of allergic-type reactions. As illustrated in this study, allergies may alter sIgA levels beyond the effects of the independent variables.

Variability in single-case data is a consistent threat to internal validity. When variability cannot be attributed to known events, the researcher can infer that there is an influence of unknown variables on the data. The many variables that exist in a study of this nature need to be better assessed and possibly controlled for with daily assessments in all pertinent areas. These include individual and collective influences on behavioral (e.g., dietary patterns, psychological and psychosocial), environmental (temperature, travel, aquatic conditions, cohabitation, etc.) and variation of sport (e.g., team sport, coacting versus interacting, coed, etc.). Also, training is not the sole source of stress in the busy lives of athletes. Life stressors aside from training need to be assessed in a more specific manner. This study assessed if there was stress present in nine domains of life, not specifically if a particular stressful event occurred and the participant's reaction to it. In doing so, variability in single-case data can be used as a mark to help identify extraneous variables to be then controlled for. 
Concerning other areas that may prove beneficial to assess as hidden variables, coping skills appear to influence an individual's ability to cope with both internal and external stressors. Athletes of comparable physical capability may differ greatly in their susceptibility to adverse training effects and overtraining. For example, there is evidence that people with greater trait anxiety may perceive any given stressor as more intense (Morgan, 1994; as cited in Hassmen, 1998). Therefore, it could be wise to assess the trait anxiety of individuals as a possible predictor variable of susceptibleness to stress.

Another important variable that could potentially influence the immune and psychological responses in women is the menstrual cycle. While there are mixed opinions, it has been suggested that menstrual and related psychological dysfunction may influence immunity in female athletes (Gleeson \& Pyne, 1998). To what extent the use of birth control pills influence the immune function in athletic populations has yet to be determined.

\section{Conclusion}

This study examined the practicality of the selected measures of mood state and immune function as markers of adverse training effects in female collegiate swimmers. Using these measures as markers of potentially adverse training effects may not cover the wide array of the unique responses that each individual may exhibit.

The findings from this investigation highlight the value of single-subject studies to monitor the psychological and biological variables of the intensely training athlete. Considerable variability was noted among participants training responses over time that would have been masked in a group experimental design. Establishing a baseline, 
monitoring frequently over the duration of the entire season, and thoroughly assessing history effects could allow for discovering a functional relation among variables in response to training. As the literature suggests, the range of individual differences in susceptibility to stress and manifestations of adverse training effects demonstrate the need to move away from group experimental designs when studying training effects in the future. 


\section{References}

Ader, R., \& Cohen, N. (1995). Psychoneuroimmunology: Interactions between the nervous system and the immune system [Electronic version]. Lancet, 345 (8942), 99- 106.

Armstrong, E., \& VanHeest, J.L. (2002). The unknown mechanism of the overtraining syndrome: Clues from depression and psychoneuroimmunology. Sports Medicine, 32, 185-209.

Barlow, D.H., \& Hersen, M. (1984). Single case experimental designs ( $2^{\text {nd }}$ ed.). Oxford: Pergamon Press.

Bagger, M., Petersen, P.H., \& Pedersen, P.K. (2003). Biological variation in variables associated with exercise training. International Journal of Sports Medicine, 24, 433-440.

Budgett, R., Newsholme, E., Lehmann, M., Sharp, C., Jones, D., \& Peto, T., et al. (2000). Redefining the overtraining syndrome as the unexplained underperformance syndrome. Sports Medicine, 34, 67-68.

Cohen, S., Tyrell, D.A., and Smith, A.P. (1991). Psychological stress and susceptibility to the common cold. New England Journal of Medicine, 325, 606-612.

Davis, M. \& Colbert, L.H. (1997). The athlete’s immune system, intense exercise, and Overtraining. In D.R Lamb \& R. Murray (Eds.), Perspectives in exercise science and sports medicine: Vol. 10. Optimizing sport performance. Carmel, IN: Cooper.

Dean, J., Whelan, J.P., \& Meyers, A.W. (1990, September) An incredibly quick way to assess mood states: The incredibly short POMS. Paper presented at the 
Association for the Advancement of Applied Sport Psychology, San Antonio, TX.

Downey, V.P. (2005) An exploration of the adjustment to college of freshmen studentathletes and non-athlete students. Unpublished Doctoral Dissertation, West Virginia University: Morgantown, WV.

Evans, P., Hucklebridge, F., \& Clow, A. (2000). Mind, Immunity, and Health: The science of psychoneuroimmunology. Free Association Books: London/ NY.

Fitzgerald, L. (1991). Overtraining increases the susceptibility to infection. International Journal of Sports Medicine, 12, S5-S8.

Foster, C. (1998). Monitoring training in athletes with reference to overtraining syndrome. Medicine and Science in Sports and Exercise, 30, 1164-1168.

Francis, J.L., Gleeson, M., Pyne, D.B., Callister, R., \& Clancy, R.L. (2005). Variation of salivary immunoglobins in exercising and sedentary populations. Medicine and Science in Sport and Exercise, 37, 571-578.

Fry, R.W., Morton, A.R., \& Keast, D. (1991). Overtraining in athletes: An update. Sports Medicine, 12, 32-65.

Gabriel, H.W., Urhausen, A., Valet, G., Heidelbach, U., \& Kindermann, W. (1998). Overtraining and immune system: a prospective longitudinal study in endurance athletes. Medicine and Science in Sport and Exercise, 30, 11511157.

Gay, L.R., \& Airasian, P. (2003). Educational research: Competencies for analysis and Applications ( $7^{\text {th }}$ ed.). Columbus, OH: Merrill Prentice Hall.

Gleeson, M. (1998). Overtraining and stress responses. Sports Exercise and Injury, 4, 
62-68.

Gleeson, M. (2002). Biochemical and immunological markers of overtraining. Journal Of Sports Science and Medicine, 1, 31-41.

Gleeson, M., Hall, S, McDonald, W., Flanagan, A., \& Clancy (1999). Salivary IgA subclasses and infection risk in elite swimmers. Immunology and Cell Biology, 77, 351-355.

Gleeson, M., McDonald, W.A., Cripps, A.W., Pyne, D.B., Clancy, R.L., \& Fricker, P.A. (1995). The effect on immunity of long term intensive training on elite swimmers. Clinical Experimental Immunology, 102, 210-216.

Gleeson, M., McDonald, W.A., Pyne, D.B., Cripps, A.W., Francis, J.L., et al. (2000) Immune status and respiratory illness for elite swimmers during a 12-week training cycle. International Journal of Sports Medicine, 21, 302-307.

Gleeson, M., McDonald, W.A., Pyne, D.B, Cripps, A.W., Francis, J.L., \& Fricker, P.A., et al. (1999). Salivary IgA levels and infection risk in elite swimmers. Medicine and Science in Sports and Exercise, 31, 67-73.

Gleeson, M., Pyne, D.B., Austin, J.P., Francis, J.F., Clancy, R.L., McDonald, W.A., \& Fricker, P.A. (2002). Epstein-Barr virus reactivation and upper respiratory tract illnesses in elite swimmers. Medicine and Science in Sport and Exercise, 34, 411-417.

Halson, S.L., Lancaster, G.I., Jeukendrup, A.E., \& Gleeson, M. (2003). Immunological responses to overreaching in cyclists. Medicine and Science in Sport and Exercise, 35, 854-861.

Hartman, U., \& Meister, J. (2000). Training and overtraining markers in selected sport 
events. Medicine and Science in Sport and Exercise, 32, 209-215.

Hassmen, P. (1998). Overtraining and recovery: A conceptual model. Sports Medicine, 26, $1622-1542$.

Hawley, C., \& Schoene, J. (2003). Overtraining syndrome. Physician \& Sportsmedicine. 31 (6), 25-32.

Henschen, K. (2000). Maladaptive fatigue syndrome and emotions in sport. In Y.L. Hanin (Eds.), Emotions in sport (pp. 191-207). Champaign, IL: Human Kinetics. Hooper, S., MacKinnon, L.T.,Gordon, R.D., \& Bachmann, A.W. (1993). Hormonal responses of elite swimmers to overtraining. Medicine and Science in Sport and Exercise, 25, 741-747.

Hooper, S., MacKinnon, L.T., Hanrahan, S. (1997). Mood states as an indication of staleness and recovery. International Journal of Sport Psychology, 28, 1-12.

Kellman, M. (2002). Psychological assessment of under recovery. In M. Kellman (Ed.) Enhancing recovery: Preventing underperformance in Athletes (pp. 3-24). Champaign, IL: Human Kinetics.

Kellman, M. (2003). Under recovery and overtraining: Different concepts- similar impact? Olympic Coach, 15 (3), 4-7.

Kennedy, C.H. (2005) Single case designs for educational research. Boston, MA: Allyn and Bacon.

Kentta, G., \& Hassmen, P.(2002). Under recovery and overtraining: A conceptual model. In M. Kellman (Ed.) Enhancing recovery: Preventing underperformance in athletes (pp. 3-24). Champaign, IL: Human Kinetics. 
Kinugasa, T., Cerin, E., \& Hooper, S. (2004). Single-subject research designs and data analyses for assessing elite athletes’ conditioning. Sports Medicine, 34, 10351050.

Lehmann, M., Foster, C., \& Keul, J. (1993). Overtraining in endurance athletes: a brief review. Medicine and Science in Sports and Exercise, 25 (7), 854-862.

MacKinnon, L.T. (1996). Immunoglobin, antibody, and exercise. Exercise Immunology Review, 2, 1-35.

MacKinnon, L.T. (1997). Immunity in athletes. International Journal of Sports Medicine, 18, S62-S62.

MacKinnon, L.T. (2000). Overtraining effects on immunity and performance in athletes. Immunology and Cell Biology, 78, 502-509.

MacKinnon, L.T., Ginn, E., \& Seymour, G.T. (1993). Decreased salivary immunoglobin-A secretion rate after intense exercise in elite kayakers. European Journal of Applied Physiology and Occupational Physiology, 67 (2), 180-184.

MacKinnon, L.T., \& Hooper, S. (1994). Mucosal (secretory) immune system responses to exercise of varying intensity and during overtraining. International Journal of Sports Medicine, 15, S179-S183.

MacKinnon, L.T., \& Hooper, S.L. (1996). Plasma glutamine and upper respiratory tract infections during intensified training in swimmers. Medicine and Science in Sport and Exercise, 28, 285-290.

Maier, S.F., \& Watkins, L.R. (1998). Cytokines for psychologists: Implications of bidirectional immune-to-brain communication for understanding behavior, 
mood, and cognition. Psychological Review, 105 (1), 83-107.

Maier, S.F., Watkins, L.R., \& Fleshner, M. (1994). Psychoneuroimmunology: The interface between brain, behavior, and immunity. American Psychologist, 49 (12), 1004-1017.

McDowell, S.L., Hughes, R.A., Hughes, R.J., Housh, D.J., Housh, T.J., \& Johnson, G.O. (1992). The effect of exhaustive exercise on salivary Immunoglobin A. The Journal of Sports Medicine and Physical Fitness, 32, 412-415.

McGrew, C.A. (1995) Exercise and Immunology. Sports Medicine and Arthroscopy Review, 3, 101-106.

McKenzie, D.C. (1999). Markers of excessive exercise. Canadian Journal of Applied Physiology, 24 (1), 66-73.

McNair, D., Lorr, M., \& Droppelman, L. (1971). Profile of Mood State manual. San Diego: Educational and Industrial Testing Service.

Morgan, W.P., Brown, D.R., Raglin, J.S., O’Connor, P.J., \& Ellickson, K.A.(1987). Psychological monitoring of overtraining and staleness. British Journal of Sports Medicine. 21 (3), 107-114.

Morgan, W.P., Costill, D.L., Flynn, M.G., Raglin, J.S., \& O’Connor, J.P. (1988) Mood disturbance following increased training in swimmers. Medicine and Science in Sports and Exercise, 20 (4), 408-414.

Neiman, D.C. (1994). Exercise, infection and immunity. International Journal of Sports Medicine, 15, S131-S141.

Neiman, D.C. (2000) Is infection risk linked to exercise workload? Medicine and Science in Sports and Exercise, 32 (7), S406-S411. 
Newsholme, E.A. (1994) Biochemical mechanisms to explain immunosupression in well- trained and over trained athletes. International Journal of Sports Medicine, 15, S142-S147.

Novas, A.M.P., Rowbottom, D.G., \& Jenkins, D.G. (2003) Tennis, incidence of URTI and salivary IgA. International Journal of Sports Medicine, 24, 223-229.

O’Connor, P.J. (1998). Overtraining and staleness. In W.P. Morgan (Ed.), Physical activity and mental health (pp.149-160). Washington, DC: Taylor \& Francis.

Otani, A. (1985). Psychometric studies of the Campus Stress Questionnaire (CSQ): A maximum likelihood factor composite technique. Unpublished Doctoral Dissertation, West Virginia University: Morgantown, WV.

Petibois, C., Cazorla, G., Poortmans, J., \& Deleris, G. (2003). Biochemical aspects of overtraining in endurance sports: The metabolism alteration process syndrome. Sports Medicine, 33 (2), 83-94.

Platen, P. (2002). Overtraining and the endocrine system - Part 2: Review of the Scientific Studies. European Journal of Sport Science, 2 (1), 1-48.

Putlur, P., Foster, C., Miskowski, J.A., Kane, M., Burton, S.E., \& Scheett, T.P., et al. (2004). Alteration of immune function in women collegiate soccer players and college students. Journal of Sports Science and Medicine, 3, 234-243.

Pyne, D.B., \& Gleeson, M. (1998). Effects of intensive exercise training on immunity in athletes. International Journal of Sports Medicine, 19, S183-S194.

Pyne, D.B., Gleeson, M., McDonald, W.A., Clancy, R.L., Perry Jr., C., \& Fricker, P.A. (2000). Training strategies to maintain immunocompetence in athletes. International Journal of Sports Medicine, 21 (supp. 1), S51-S60. 
Robson, P.J. (2003). Elucidating the unexplained underperformance syndrome in endurance athletes: The Interleukin-6 hypothesis. Sports Medicine, 33 (10), 771781.

Salimetrics. LLC. (2005). Collection advice: Salivary samples from human subjects. [Brochure]. State College, PA: Author.

Shephard, R.J., \& Shek, P.N. (1994). Potential impact of physical activity and sport on the immune system: a brief review. British Journal of Sports Medicine, 28, 347355.

Shephard, R.J., Shek, P.N. (1998). Acute and chronic overexertion: Do depressed immune responses provide useful markers? International Journal of Sports Medicine, 19, 159-171.

Smith, L.L. (2000). Cytokine hypothesis of overtraining: a physiological adaptation to excessive stress? Medicine and Science in Sport and Exercise, 32, 317-331.

Smith, L.L. (2003). Overtraining, excessive exercise, and altered immunity: Is this a T Helper-1 versus T Helper-2 lymphocyte response? Sports Medicine, 33, 347364.

Smith, D.J., \& Norris, S.R. (2002). Training load and monitoring an athlete’s tolerance for endurance training. In M. Kellman (Ed.), Enhancing recovery: Preventing underperformance in athletes (pp. 3-24). Champaign, IL: Human Kinetics.

Tharp, G.D. (1991). Basketball exercise and secretory immunoglobin A. European Journal Of Applied Physiology, 63, 312-314.

Tharp, G.D. \& Barnes, M.W. (1990). Reduction of saliva immunoglobin levels by swim training. European Journal of Applied Physiology, 60, 61-64. 
Tiollier, E., Gomez-Merino, D., Burnat, P., Jouanin, J.-C., Bourrilhon, C., Filaire, E., et al. (2005). Intense training: mucosal immunity and incidence of respiratory infections. European Journal of Applied Physiology, 93, 421-428.

Urhausen, A., \& Kindermann, W. (2002). Diagnosis of overtraining: What tools do we have? Sports Medicine, 32, 95-102. 
Table 1

Means and Standard Deviations of Mood State, Life Stress and sIgA Concentrations

Participant

\begin{tabular}{|c|c|c|c|c|c|c|c|c|c|c|}
\hline & \multicolumn{2}{|c|}{$\mathrm{P} 1$} & \multicolumn{2}{|c|}{ P2 } & \multicolumn{2}{|c|}{ P3 } & \multicolumn{2}{|c|}{$\mathrm{P} 4$} & \multicolumn{2}{|c|}{ P5 } \\
\hline & Mean & $\underline{\mathrm{SD}}$ & Mean & $\underline{\mathrm{SD}}$ & Mean & $\underline{\mathrm{SD}}$ & Mean & $\underline{\mathrm{SD}}$ & Mean & $\underline{\mathrm{SD}}$ \\
\hline Mood State & 10 & 3.36 & 3.45 & 4.46 & 3.43 & 3.78 & 3.33 & 2.65 & 3.14 & 4.26 \\
\hline Life Stress & 31.43 & 3.15 & 21.71 & 6.29 & 9.29 & 3.25 & 13.83 & 4.21 & 10.43 & 6.48 \\
\hline $\begin{array}{l}\text { sIgA } \\
\text { Concentration }\end{array}$ & 389.29 & 227.23 & 450.13 & 123.36 & 1162.84 & 828.19 & 340.74 & 344.97 & 305.011 & 18.36 \\
\hline
\end{tabular}

Note. Mood state was measured by the BAM on a 5-point Likert scale ranging from 0 (not at all) to 4 (extremely). Life stress was measured by a short stress questionnaire on a 9-point Likert scale ranging from 0 (none) to 9 (extreme). Mood state and life stress are calculated with a total score, in which higher values indicate greater mood disturbance and life stress. sIgA concentration was measured in units ug/mL of saliva. 
Table 2

Bivariate Correlations with Test Variables For Participants

\begin{tabular}{|c|c|c|c|}
\hline Participant 1 & $\begin{array}{l}\text { Mood } \\
\text { State } \\
\end{array}$ & $\begin{array}{l}\text { Life } \\
\text { Stress } \\
\end{array}$ & $\begin{array}{c}\text { sIgA } \\
\text { Concentration } \\
\end{array}$ \\
\hline Mood State & 1 & .078 & .525 \\
\hline Life Stress & & 1 & -.212 \\
\hline sIgA Concentration & & & 1 \\
\hline \multicolumn{4}{|l|}{ Participant 2} \\
\hline Mood State & 1 & -.622 & -.707 \\
\hline Life Stress & & 1 & .669 \\
\hline sIgA Concentration & & & 1 \\
\hline \multicolumn{4}{|l|}{ Participant 3} \\
\hline Mood State & 1 & .504 & -.213 \\
\hline Life Stress & & 1 & -.056 \\
\hline sIgA Concentration & & & 1 \\
\hline \multicolumn{4}{|l|}{ Participant 4} \\
\hline Mood State & 1 & -.226 & .355 \\
\hline Life Stress & & 1 & .036 \\
\hline sIgA Concentrations & & & 1 \\
\hline \multicolumn{4}{|l|}{ Participant 5} \\
\hline Mood State & 1 & $.891^{* *}$ & -.298 \\
\hline Life Stress & & 1 & .449 \\
\hline sIgA Concentration & & & 1 \\
\hline
\end{tabular}

Note. Numbers represent Pearson Correlation Coefficients. ** Correlation is significant at the .01 level. 
Training: Effects on Immunity and Mood - 39 -

Figure 1. Individual training load per week for each participant, measured in yards over seven weeks.

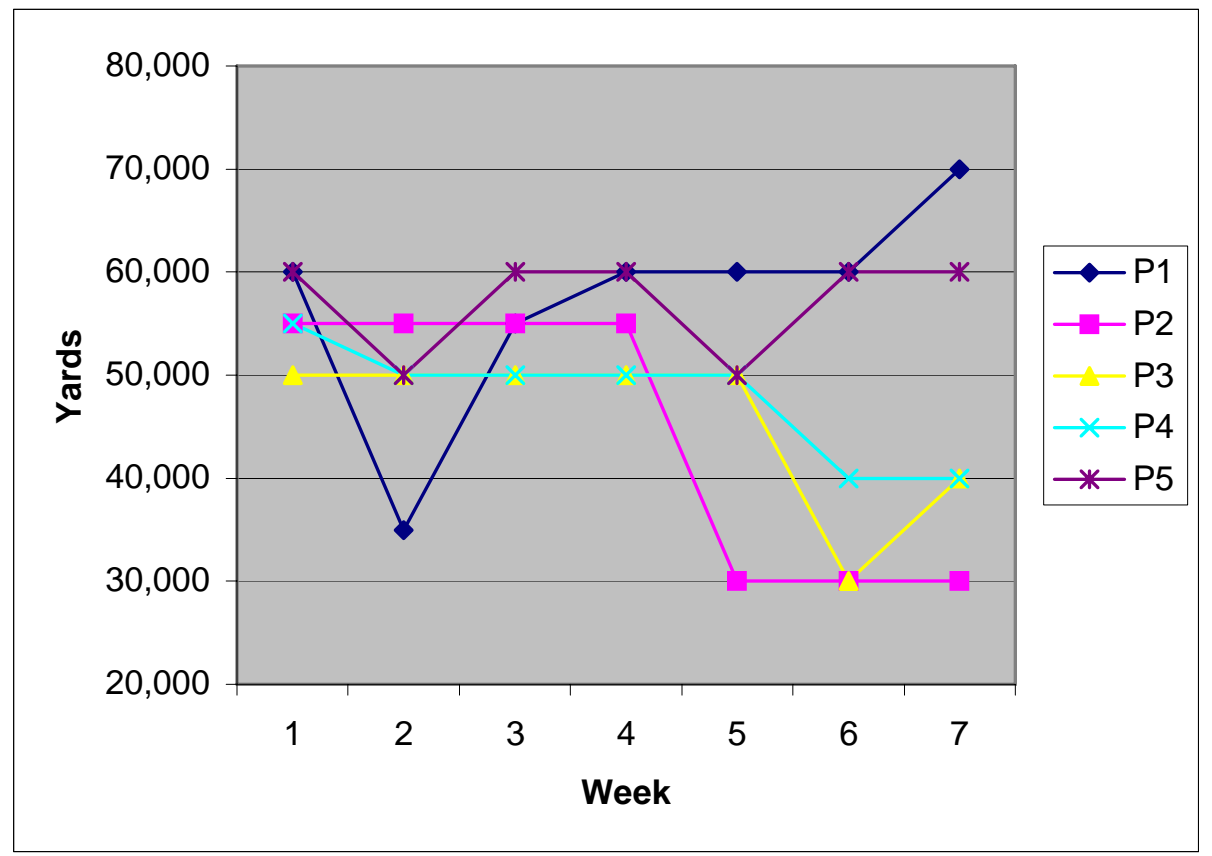


Figure 2. Mood, as measured by the BAM, and life stress, as measured by the short stress questionnaire, over 7 weeks for P1.

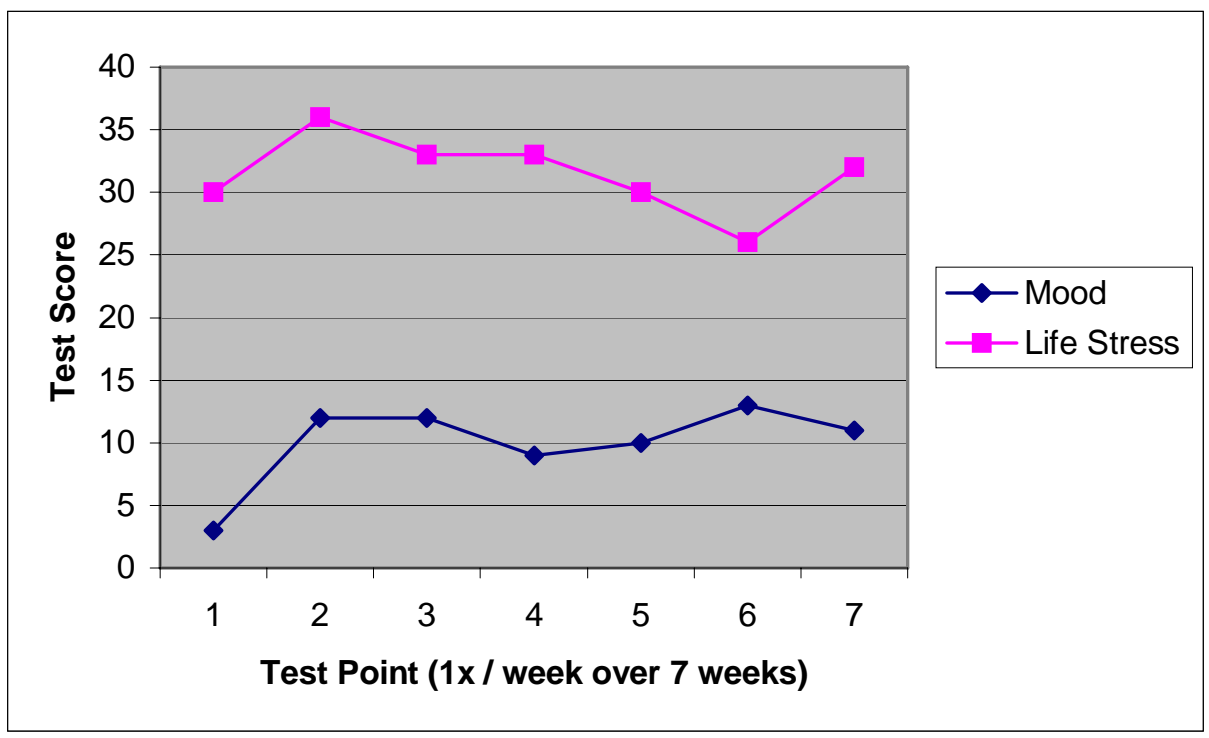


Figure 3. Salivary IgA concentration in ug/mL, measured once per week over seven weeks for P1.

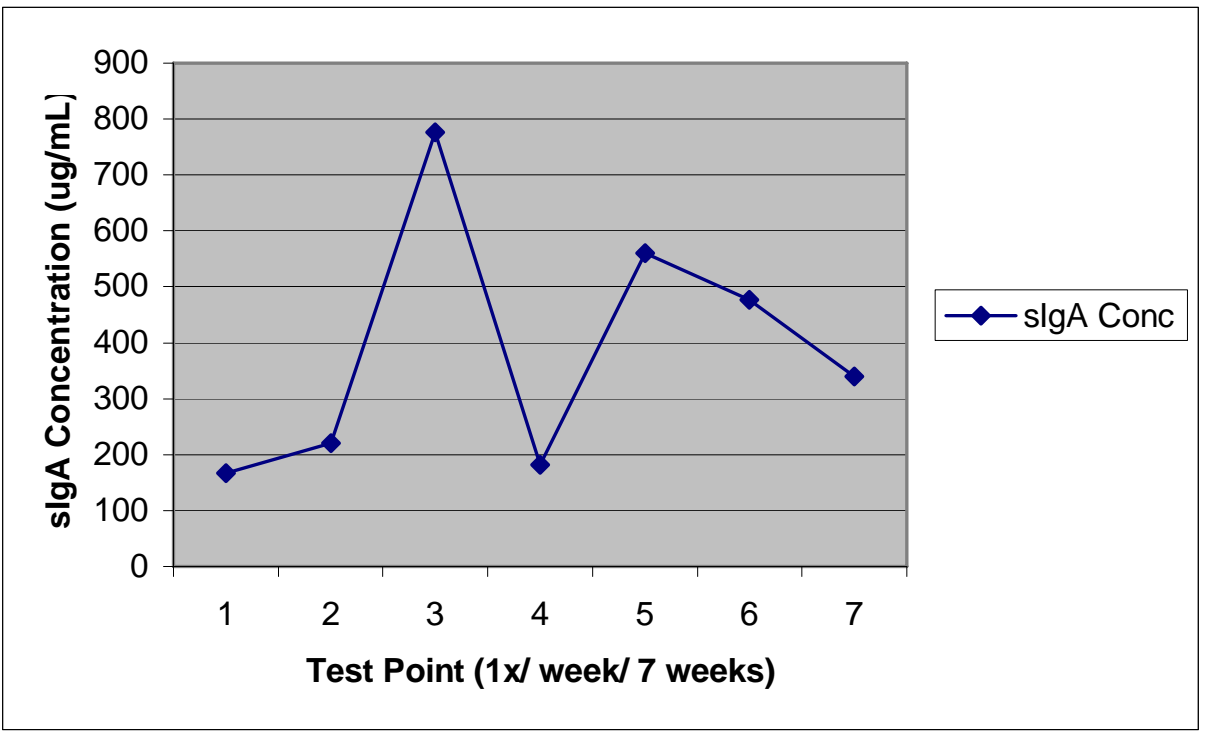


Figure 4. Mood, as measure by the BAM, and life stress, as measured by the short stress questionnaire, over three consecutive days during Week 1 for P1.

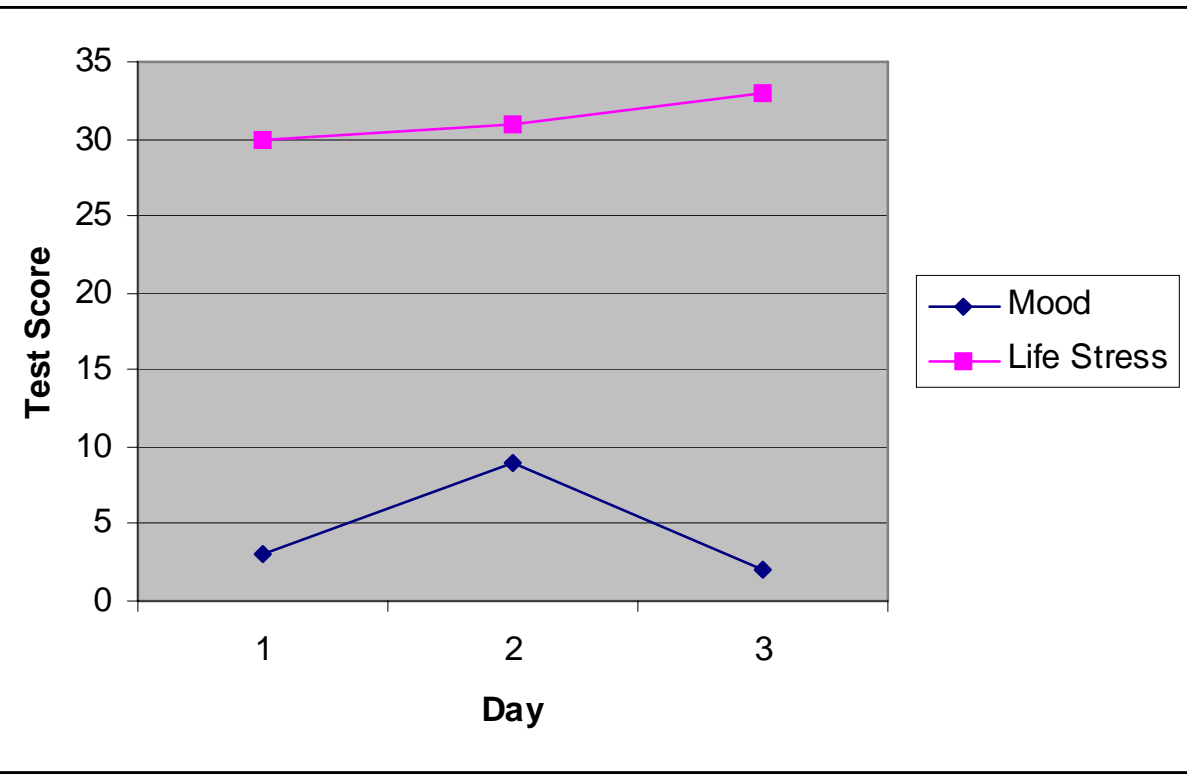


Figure 5. Mood, as measured by the BAM, and life stress, as measured by the short stress questionnaire, over 7 weeks for P2.

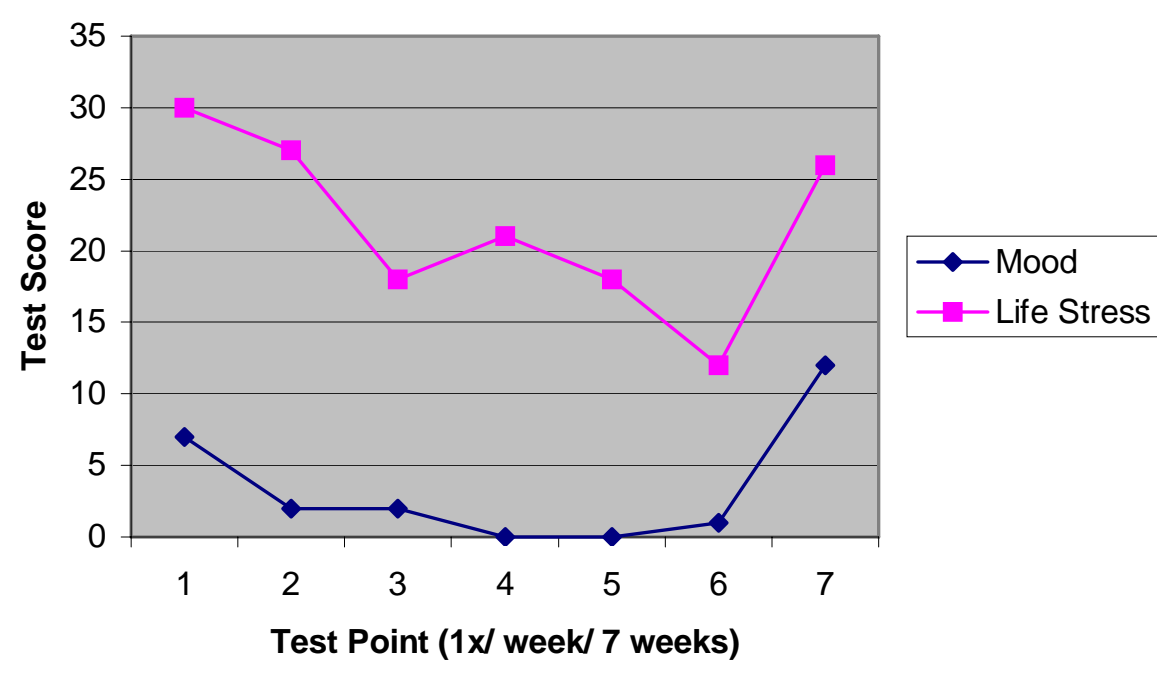


Figure 6. Salivary IgA concentration in ug/mL, measured once per week over seven weeks for P2.

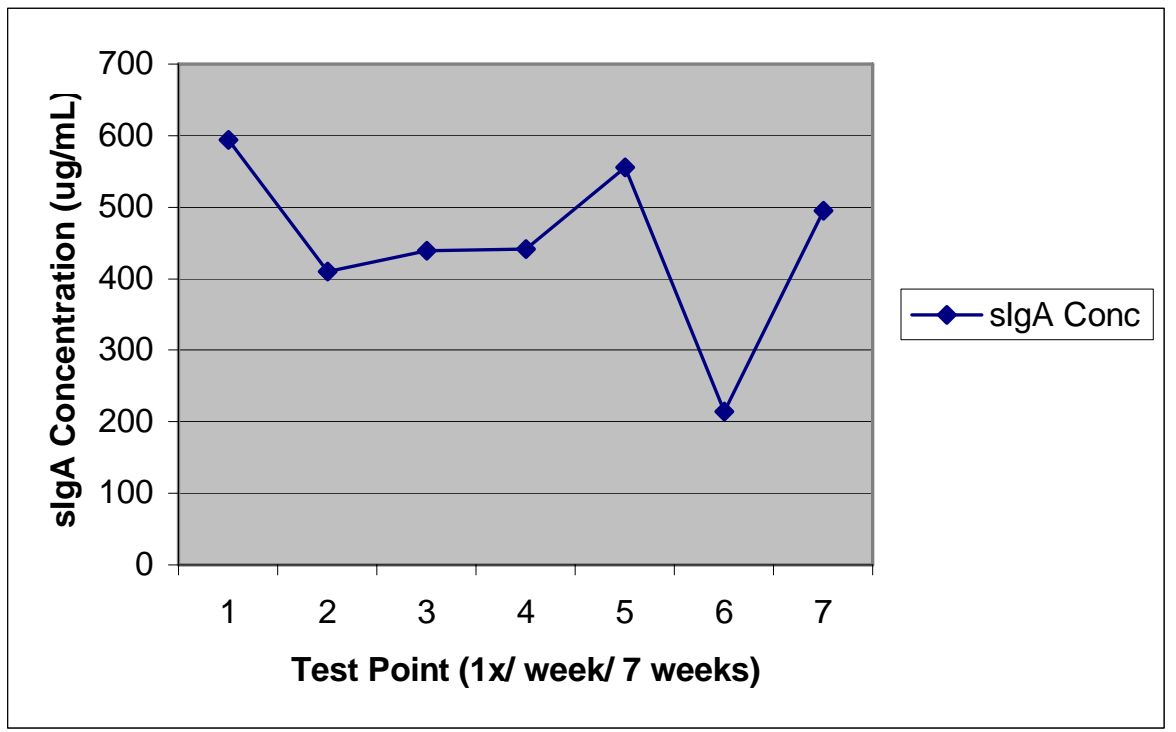


Figure 7. Mood, as measure by the BAM, and life stress, as measured by the short stress questionnaire, over three consecutive days during Week 1 for P2.

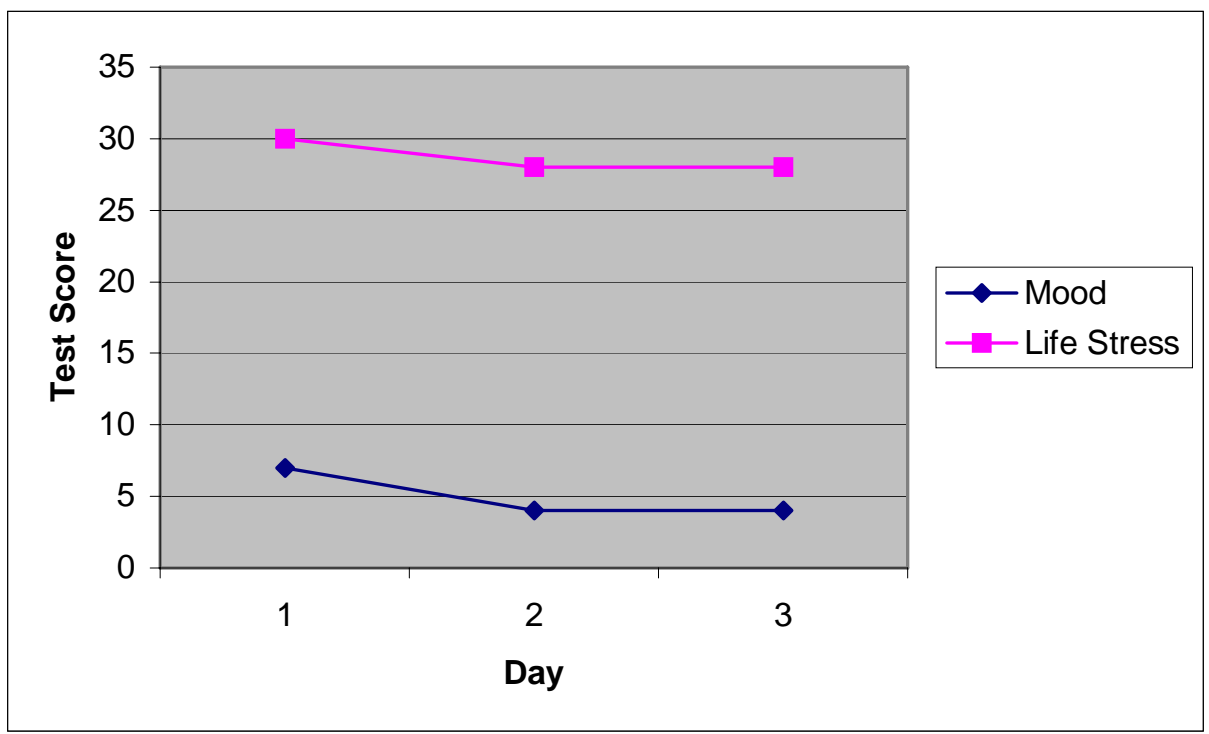


Figure 8. Salivary IgA concentration in ug/mL, over three consecutive days during Week 1 for P2.

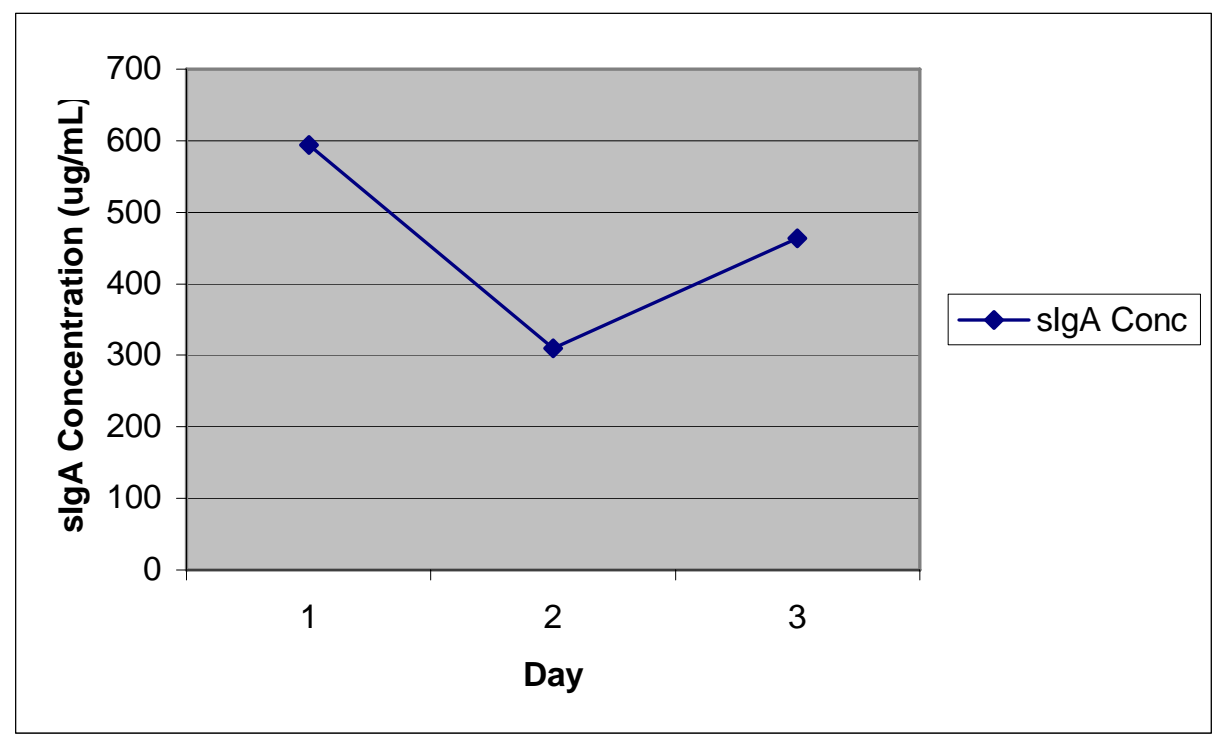


Training: Effects on Immunity and Mood - 47 -

Figure 9. Mood, as measured by the BAM, and life stress, as measured by the short stress questionnaire, over 7 weeks for P3.

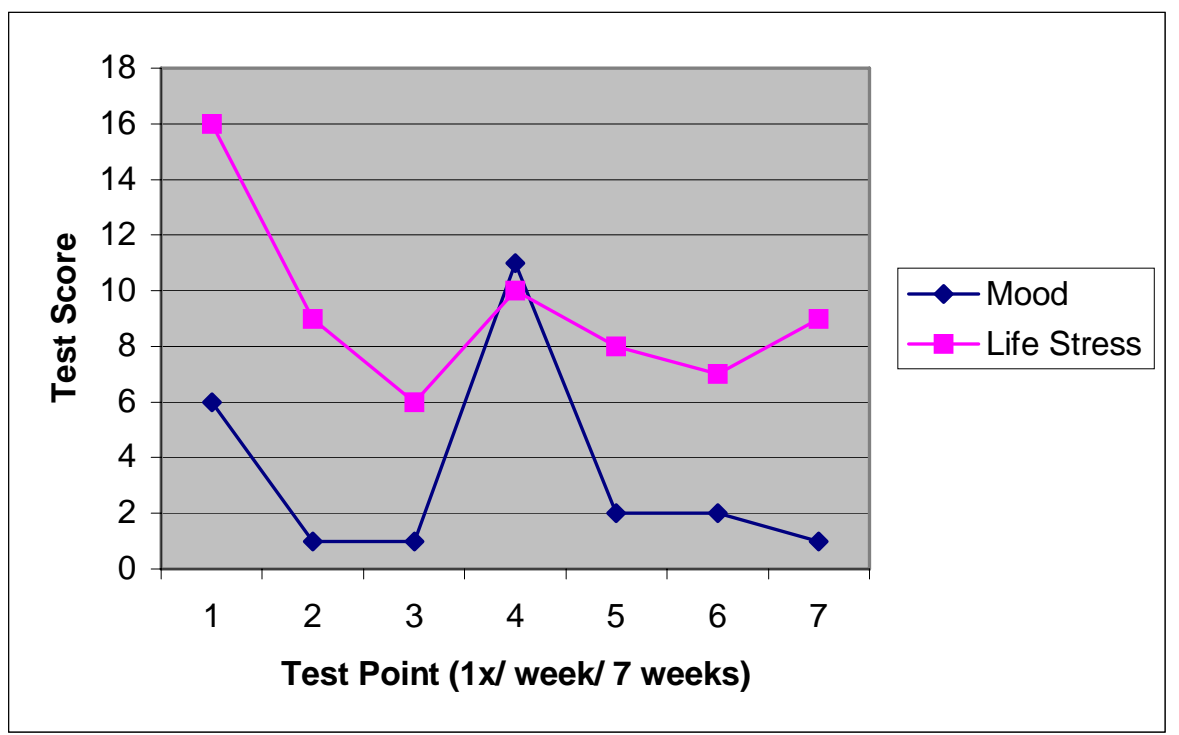


Figure 10. Salivary IgA concentration in ug/mL, measured once per week over seven weeks for P3.

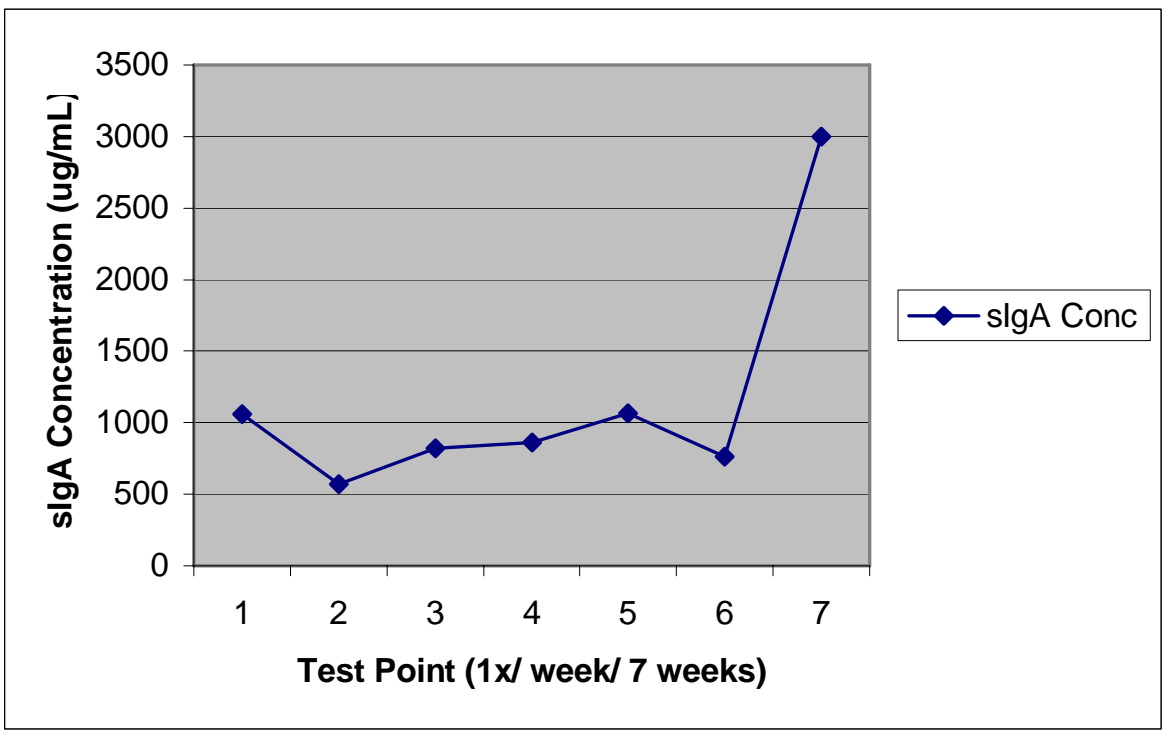


Figure 11. Mood, as measure by the BAM, and life stress, as measured by the short stress questionnaire, over three consecutive days during Week 1 for P3.

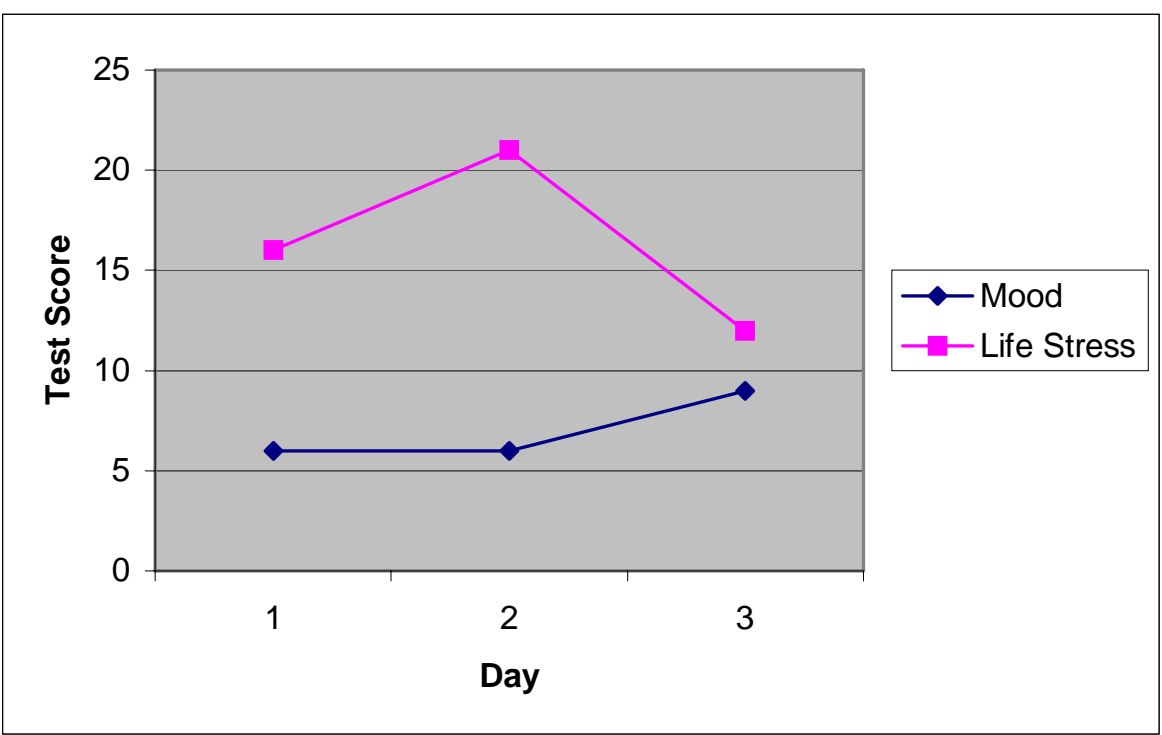


Training: Effects on Immunity and Mood - 50 -

Figure 12. Salivary IgA concentration in ug/mL, over three consecutive days during Week 1 for P3.

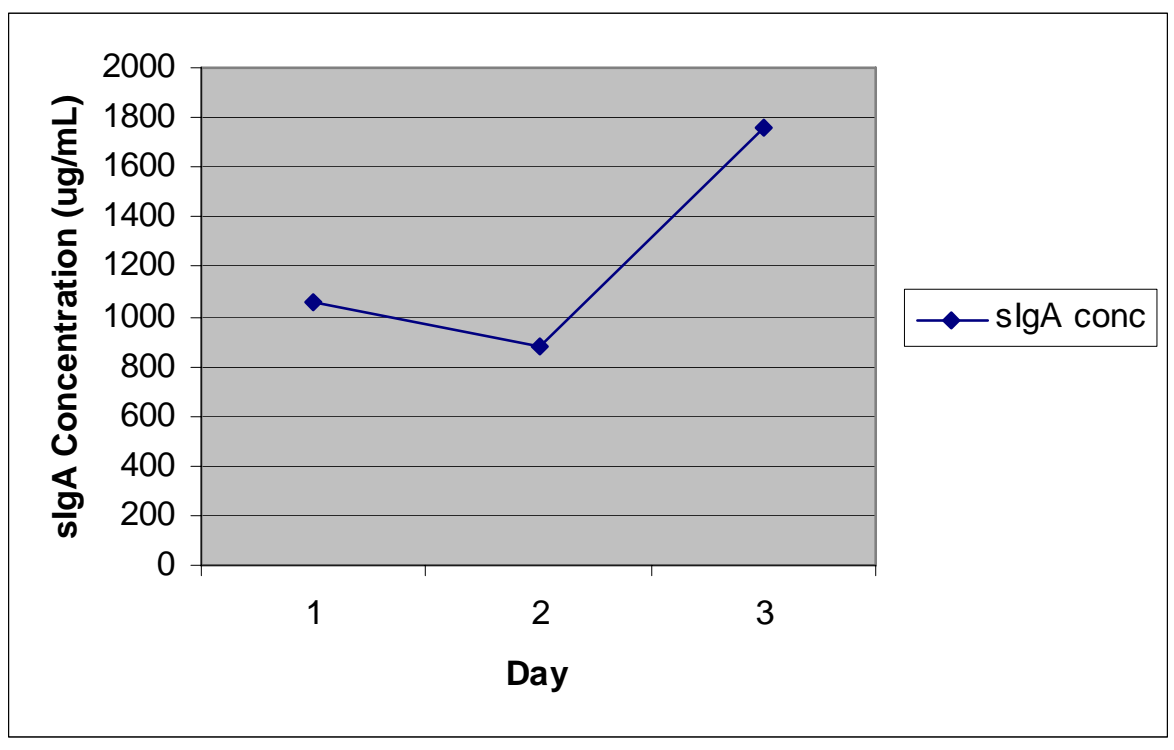


Figure 13. Mood, as measured by the BAM, and life stress, as measured by the short stress questionnaire, over 7 weeks for P4.

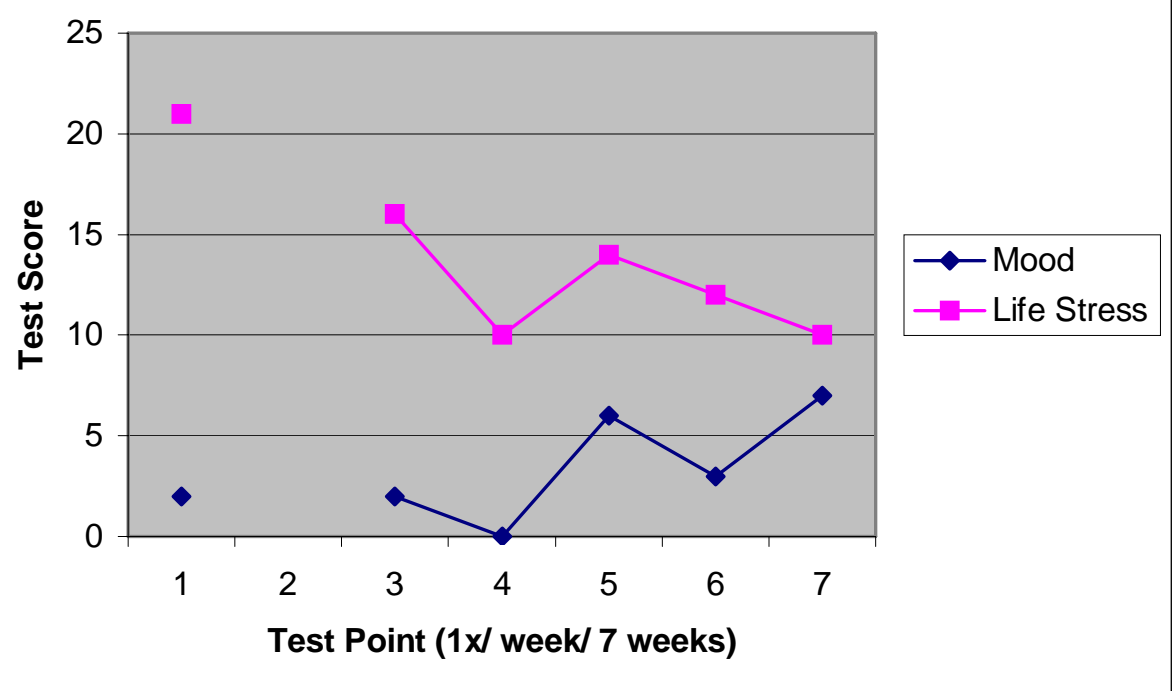


Training: Effects on Immunity and Mood - 52 -

Figure 14. Salivary IgA concentration in ug/mL, measured once per week over seven weeks for P4.

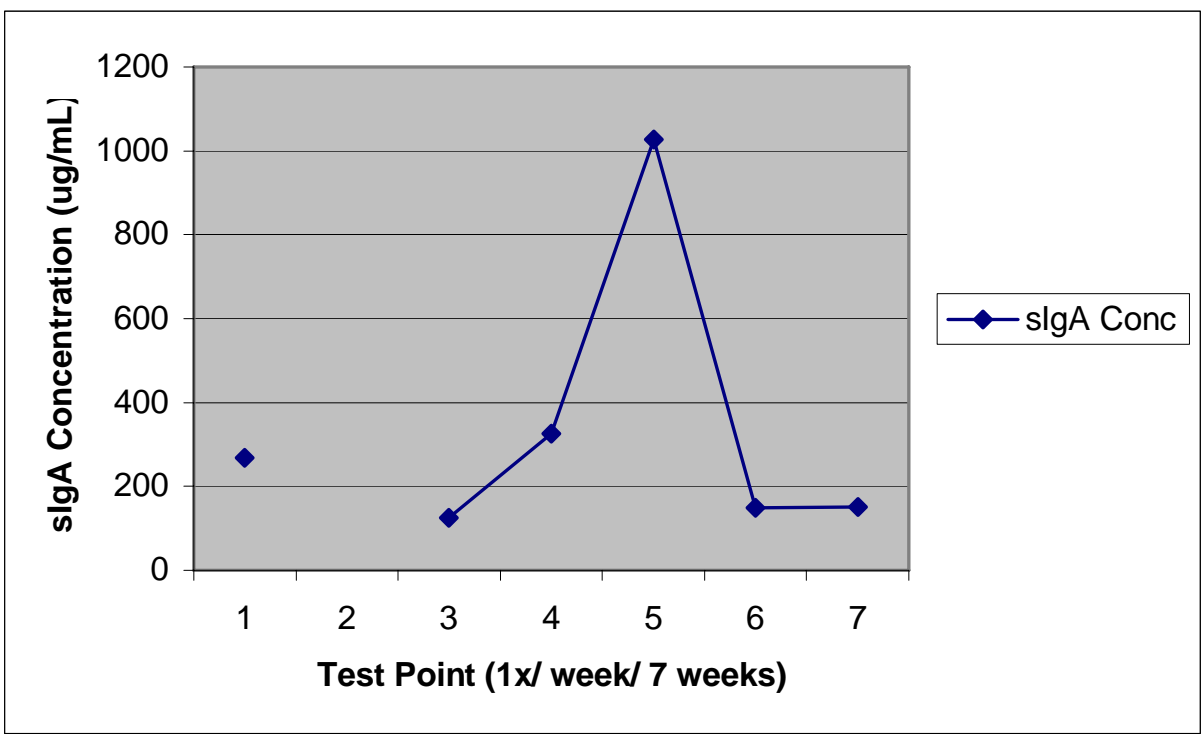


Figure 15. Mood, as measure by the BAM, and life stress, as measured by the short stress questionnaire, over three consecutive days during Week 1 for P4.

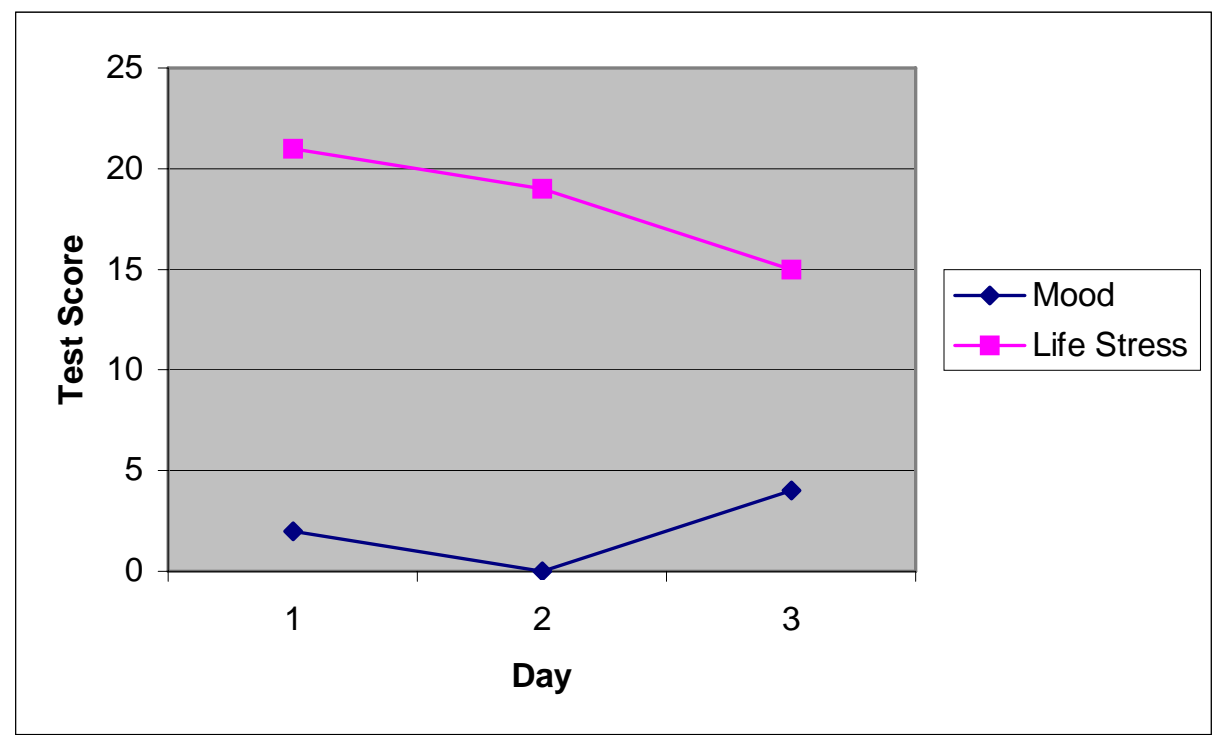


Training: Effects on Immunity and Mood - 54 -

Figure 16. Salivary IgA concentration in ug/mL, over three consecutive days during Week 1 for P4.

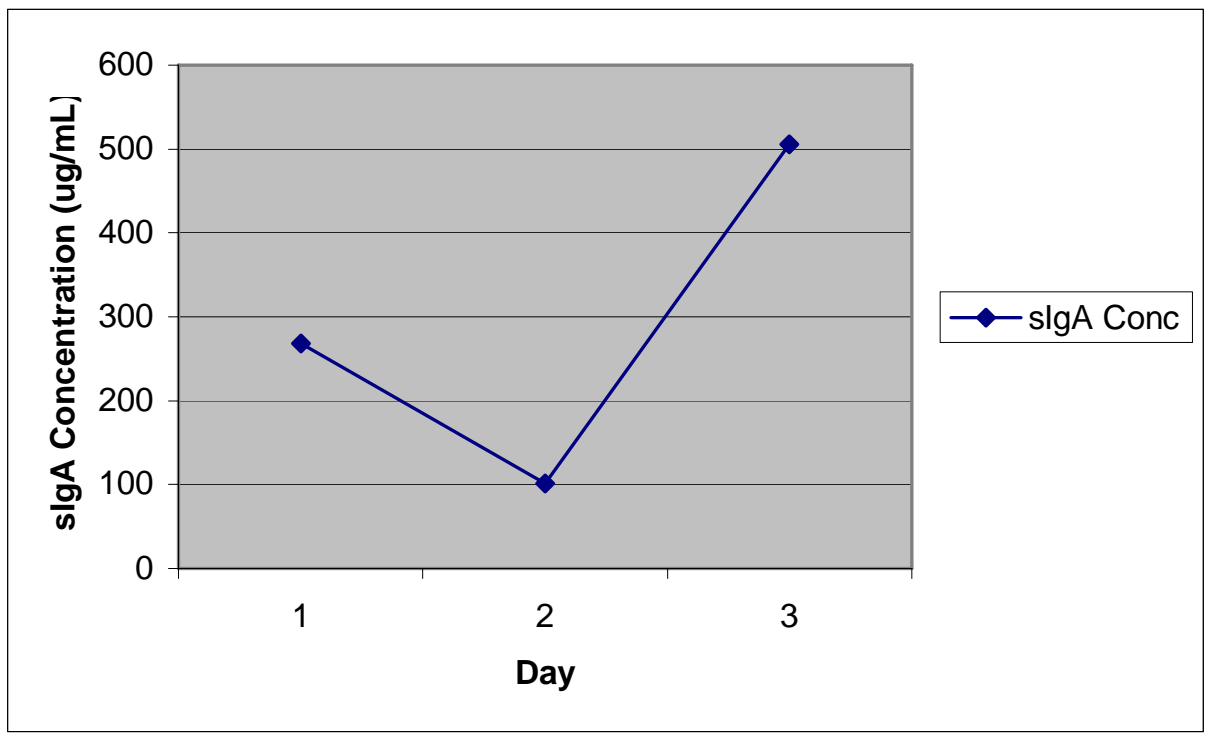


Figure 17. Mood, as measured by the BAM, and life stress, as measured by the short stress questionnaire, over 7 weeks for P5

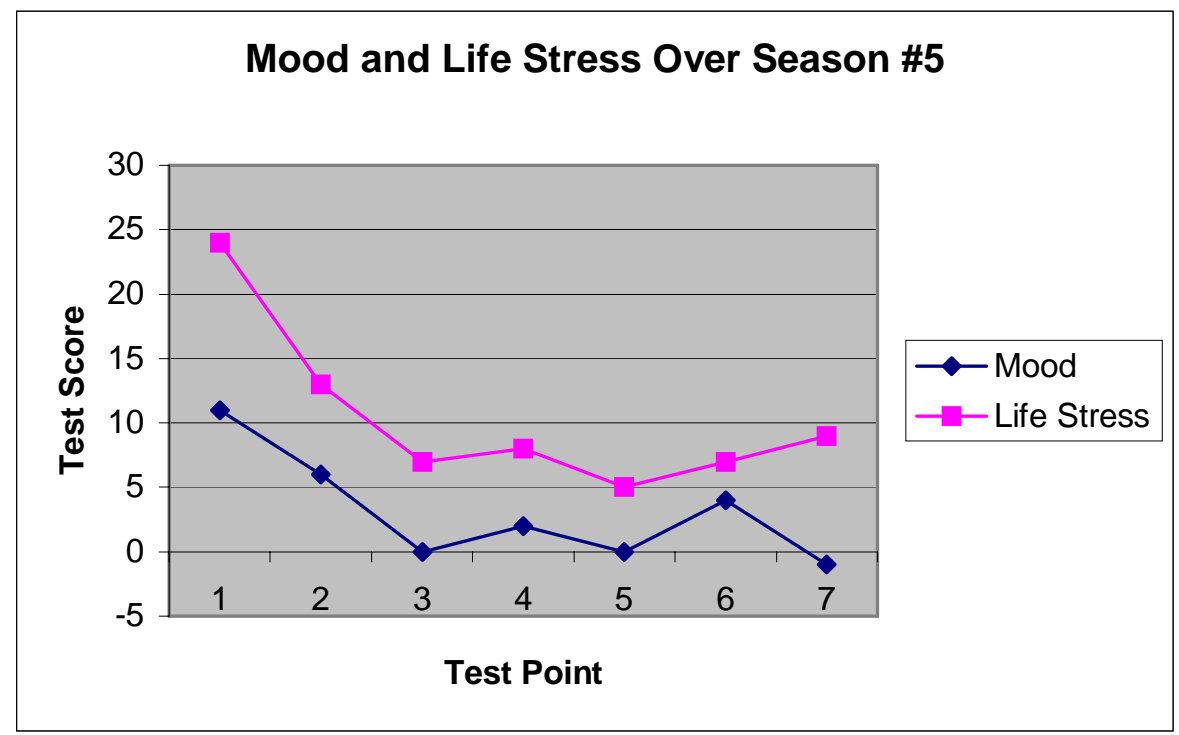


Training: Effects on Immunity and Mood - 56 -

Figure 18. Salivary IgA concentration in ug/mL, measured once per week over seven weeks for P5.

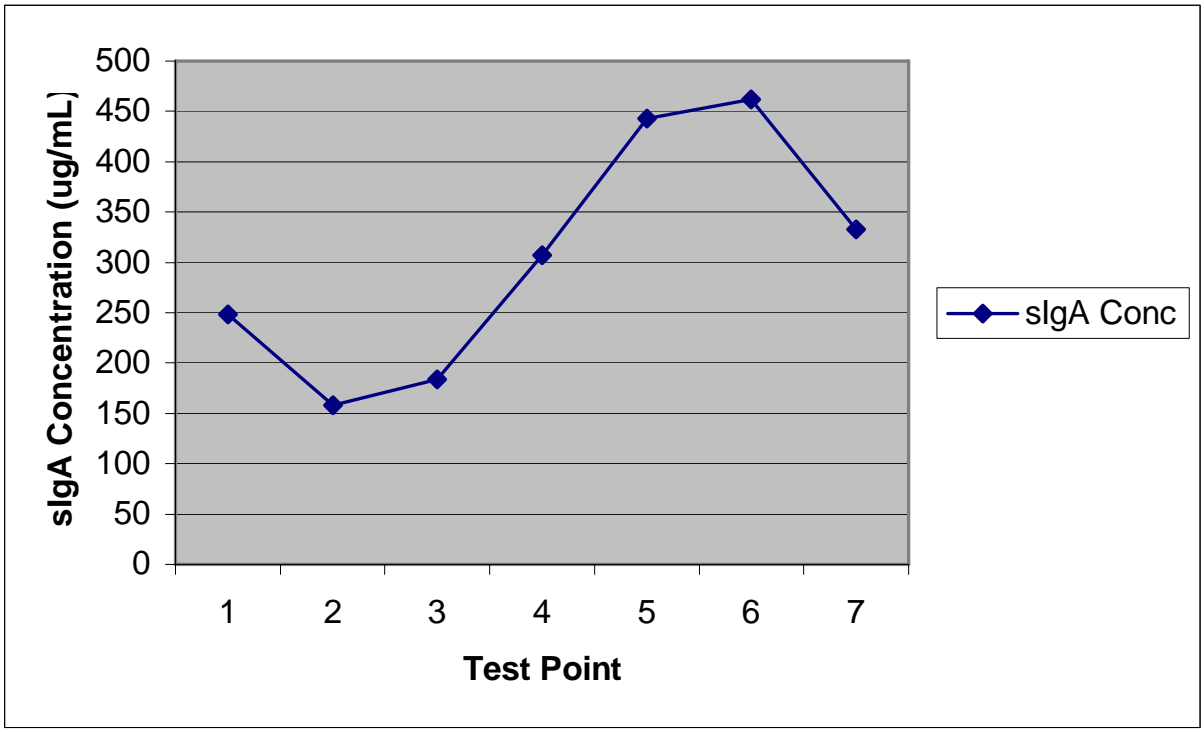


Figure 19. Mood, as measure by the BAM, and life stress, as measured by the short stress questionnaire, over three consecutive days during Week 1 for P5.

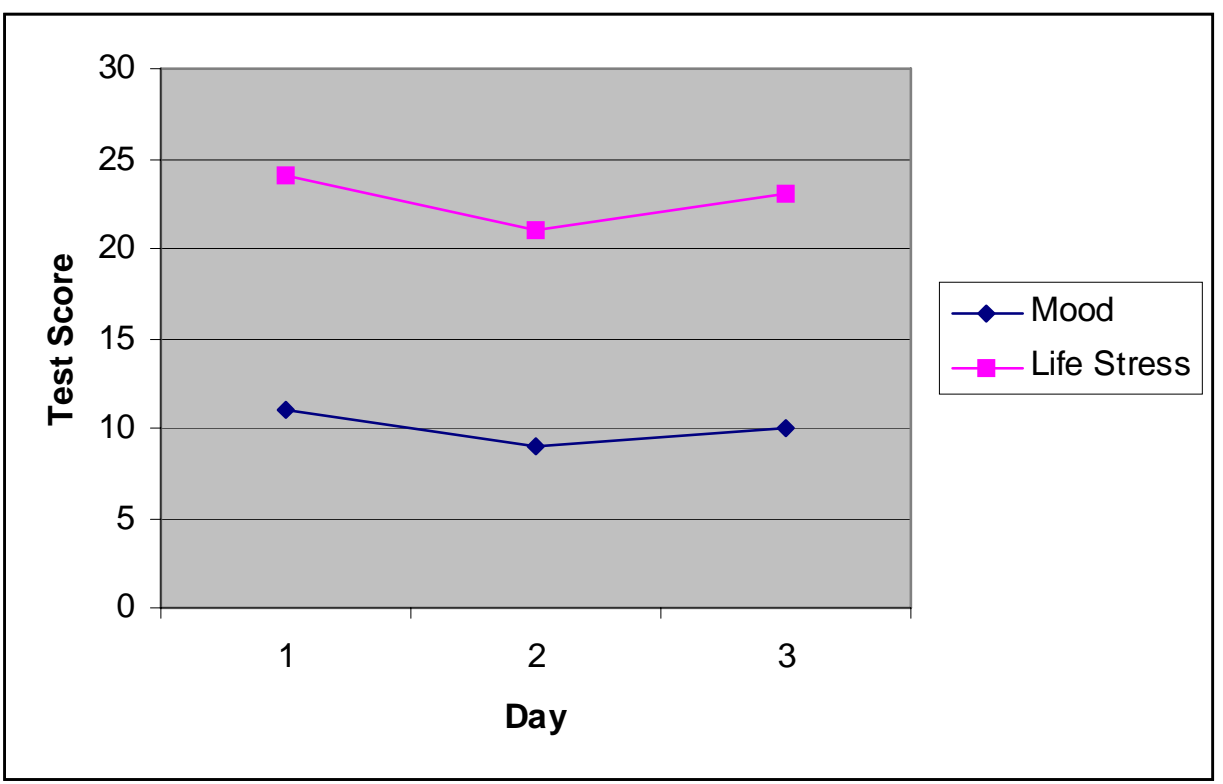


Training: Effects on Immunity and Mood - 58 -

Figure 20. Salivary IgA concentration in ug/mL, over three consecutive days during Week 1 for P5.

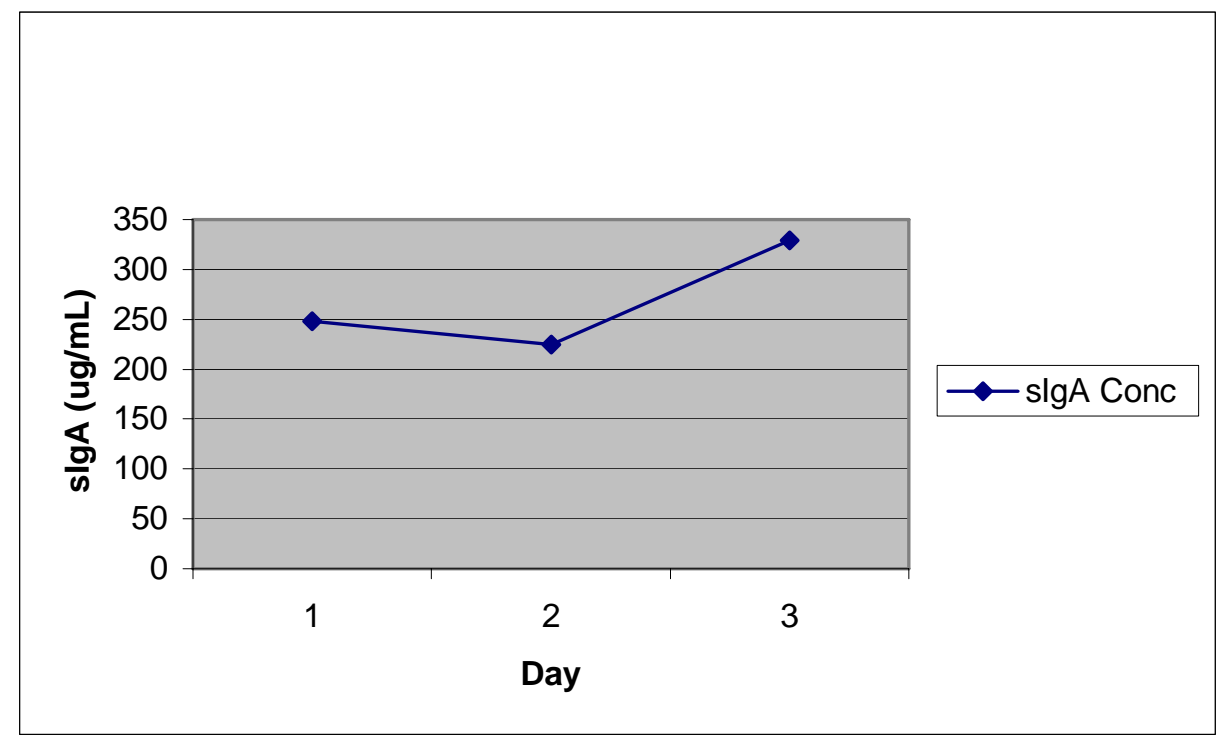




\section{Appendix A}

Brief Assessment of Mood State Questionnaire

Reprinted with permission from Whelan and Meyers (1990)

Brief Assessment of Mood

Circle the number that best describes how you are feeling right now.

Not at all A little Moderately Quite a bit Extremely

How anxious do you feel?

$\begin{array}{lllll}0 & 1 & 2 & 3 & 4\end{array}$

How sad or depressed do you feel?

0

1

2

3

4

How confused do you feel?

How angry do you feel?

0

1

2

3

4

How energetic do you feel?

0

1

2

3

4

How fatigued do you feel?

12

3

4

$\begin{array}{llll}1 & 2 & 3 & 4\end{array}$




\section{Appendix B}

Training Load/ Illness Assessment

1) How many yards did you swim in the past week?

2) In the past week, have you experienced any respiratory infections, defined as symptoms of fever, sore throat, tonsillitis, stuffy nose and swollen glands consistent with either overt bacterial infection or viral illness (Gleeson, Hall, et al., 1999)? If yes, please briefly specify. 


\section{Appendix C}

\section{Short Stress Questionnaire}

The following are statements about typical factors that can cause stress in the life of a college athlete. Please circle the degree to which the following seven factors have caused stress in your life within the past week using the anchors of (1) as having little stress and (8) as having extreme stress. Use the number 0 if that factor has not caused any stress in your life in the past week.

\section{None Little}

Moderate

Extreme

\begin{tabular}{|l|l|l|l|l|l|l|l|l|l|}
\hline 1. Academic factors & 0 & 1 & 2 & 3 & 4 & 5 & 6 & 7 & 8 \\
\hline 2. Personal-social factors & 0 & 1 & 2 & 3 & 4 & 5 & 6 & 7 & 8 \\
\hline 3. Financial factors & 0 & 1 & 2 & 3 & 4 & 5 & 6 & 7 & 8 \\
\hline 4. Family factors & 0 & 1 & 2 & 3 & 4 & 5 & 6 & 7 & 8 \\
\hline 5. Self-image factors & 0 & 1 & 2 & 3 & 4 & 5 & 6 & 7 & 8 \\
\hline 6. Health factors & 0 & 1 & 2 & 3 & 4 & 5 & 6 & 7 & 8 \\
\hline 7. Athletic factors & 0 & 1 & 2 & 3 & 4 & 5 & 6 & 7 & 8 \\
\hline
\end{tabular}




\title{
Appendix D
}

\section{Approval from Institutional Review Board for the Protection of Human Rights}

\author{
WestVúrginiaUniversity \\ Office of Research Compliance \\ DATE: Octuber 6,2005
}

This research will be monitorec for re-approval annually,

APPROVA HERIOD: October $6,2005=0$ Jctober 5,2006

NOTICE OF APPROVAL FOR PROTOCOL: IRB $\$ 16694$

To: Michelle 3artlett

TITLE: Intense Training in Sport: Effects on Immune Function and Mood Stal.e

AGENCY: N/A

The Tnstitutional Review Board for the Protection of Human Research subjects (IRB) has approved the projec: doscribed

above. Approval was based on the doscriptive material and procedures you submitted for review. Should any changes in your protocol/consent form be recessary, prior approval must be obtained from the IRB.

According to the Coce of Eederal Regulations, Section 312.32, investigators are required to notify the FDA and the study sponsor of any adverse experience agsocialed with the use of an investigational drug that is serious and unexpected. A

serious adverse experience is considered any event that is fatal or life-threatening, is permanently disabling, reçuires inpatient hospitalization, or is a congonital anomaly, cancer, or overdose. An unexpected adverse experience is an event that $t s$ not identified in nature, severity, or frequency in the current invest-gazor brochure. Any experience reportable to FDA and the sponsor must also be reported immediatcly to the IRB. If the stuay is funded, initiation of the protocol may not begin until the contract is finalized. 


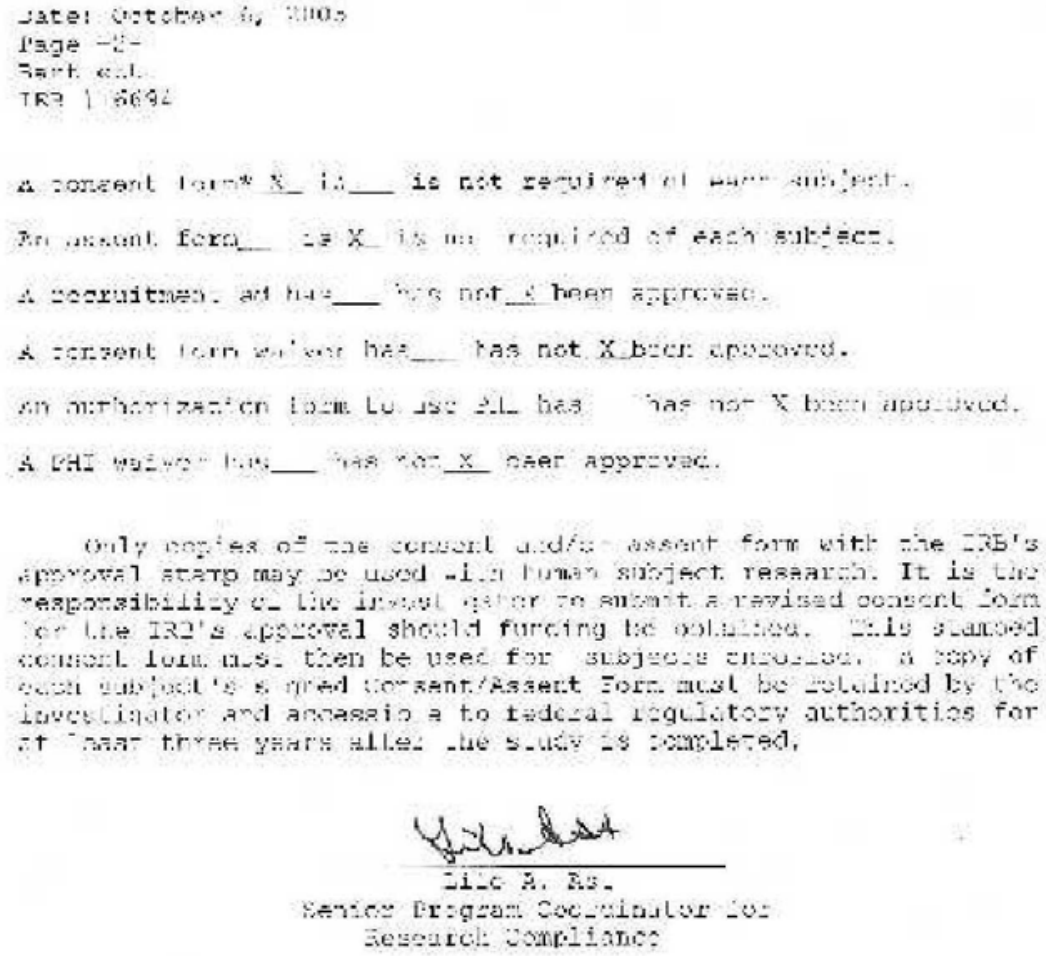




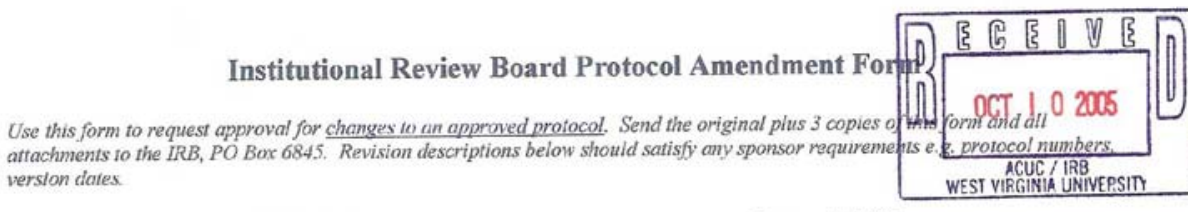

Principal Investigator: Michelle Bartlett Date: $10 / 10 / 05$

IRBA: 16694 And. I Requesting FB: $\frac{}{\text { (1 original \& 18 Copies) }}$ Exp: $-\frac{X}{\text { (1 original \& } 1 \text { copy) }}$

Protocol Title* Intense Training in Sport: Effects on Immune Function and Mood State

* The protocol title should include the version and date for all addendums, investigator brochures, etc.

Revision or Addition Description - check $(X)$ all that apply:
$X \quad$ Revision to currently approved protocol

$\begin{array}{llll}\mathrm{X} & \begin{array}{l}\text { Revision to currently approved protocol } \\ \text { Revision to currently approved consent }\end{array} & \begin{array}{l}\text { New version date: } \\ \text { New version date: } \\ \text { Revision to investigator brochure }\end{array} & \begin{array}{l}\text { New version date: } \\ \text { New version date: }\end{array}\end{array}$

Check $(\mathrm{X})$ all that apply: (For a definition of major and minor changes sec Chapter V. scetion A)

Revision involves major changes

$\mathrm{X} \quad$ Revision involves minor changes

Describe changes. Explain in detail in the space below the reasons for requesting these changes and which part(s) of the approved protocol will be amended. For all required submissions see Chapter V. section B.

-The West Virginia University Women's Swimming team will now be the participants in this study. Due to time constraints, the original participants- the West Virginia University Women's Cross Country team-are too far into their scason to be participants in this study now.

-Setting data collection will now take place in the West Virginia University Women's Swimming team's locker room.

-The Short Stress Qucstionnaire will be administered at all testing points, not just the first and last as was in the original protocal. This is being added at every testing point to more reliably gauge the effects that life stressors may have on the two dependent variables- immune function and mood state.

Describe changes to the consent form/assent form/recruitment ad. Explain which scctions of these items are being changed.

Changes to the consent form and cover letter arc: all mention of the West Virginia University Women's Cross Country team in the documents will be amended to state the West Virginia University Women's Swimming Team (section: purposes of the study- pg. 1/3, description of procedures- pg. 1/3; in the description of procequweesipg. 1/3) it will be amended to state that the Short Stress Questionnaire will be administered at every tes. mood state questionnaire).

Changes to the cover letter:

OCT 132005

TPPDOVF,

Principal or Co-Investigator

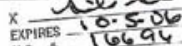

$10 / 10 / 05$

Sign below only if there are ncw or changed co-investigator(s)

Ncw Co-Investigntor(s): [typed name and signature] 


\section{Appendix E}

\section{Consent and Information Form}

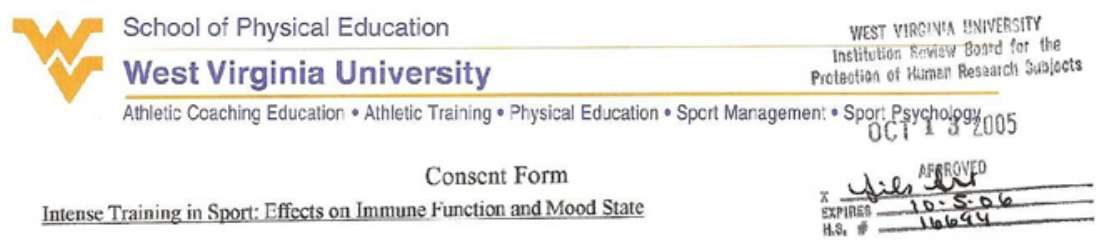

Introduction.

have been asked to participate in this research study, which has been

explained to me by the primary researcher, Michelle Bartlett, B.S. This study is being conducted by

Michelle Bartlett, B.S. in the School of Physical Education at West Virginia University.

This research is being conducted to fulfill the requirements for a master's thesis in sport and

exercise psychology in the School of Physical Education at West Virginia University, under the

supervision of Edward Etzel, Ed.D.

Purposes of the Study.

I have been told that the purpose of this study is to learn more about the relationship between intense training and immunity and mood. There will be approx, 8 participants in the study, all being members of the West Virginia University women's swimming team.

\section{Description of Procedures.}

This study will be conducted at West Virginia University. The researcher will ask me to fill out three forms early in the competitive season regarding demographics, mood state and life stressors. A saliva sample will also be requested from me for the purpose of analyzing salivary immunoglobin levels for a general gauge of Immune function. Saliva (passive drool) will be collected in a $2.0 \mathrm{ml}$ vial, sealed tightly and stored in a non-cycling freezer at ${ }^{-} 60^{-} 80^{\circ} \mathrm{C}$. Throughout the season when Michelle Bartlett meets with the women's swimming team for scheduled data collection, I will provide a saliva sample, fill out the mood state questionnaire, a short stress questionnaire and document my training load and incidence of upper respiratory tract infections a total of 8 more time times throughout the season. It will take about 10-15 minutes to answer the questions and give a saliva sample on the testing points. I understand that I may see the questions before signing the page. I do not have to answer all the questions.

Risks and Discomforts.

Some of the questions inquire about personal information, which may cause minor cognitive distress. I understand that it is my decision on whether or not I choose to complete those items.

Alternatives.

I do not have to participate in this study. Alternatives that could be considered in this case include not participating in this study.

Benefits.

I understand that this study may help expand the current knowledge of monitoring the effects of training on the immune system and mood state. It is possible that I may not gain any direct benefit from participating in this study. 
Training: Effects on Immunity and Mood - 66 -

\author{
W/r West VirginiaUniversity \\ School of Physical Education
Intense Traming in Sport: Eurecis on limmine Function and Mood State
}

Financial Considerations.

No payments will be made for participating in the study.

\begin{abstract}
Confidentiality kept as confidential as legally possible. Research records and test results, just like hospital
\end{abstract} records, may be subpoenaed by court order or may be inspected by federal regulatory authorities without my additional consent.

In addition, there are certain instances where the researcher is legally required to give information to the appropriate authorities. These would include manclatory reporting of infectious diseases, mandatory reporting of information about behavior that is imminenly dangerous to me or to others, such as suicide, child abuse, etc.

Ali data sources will be kept locked up and will be destroyed as soon as possible after the research is finished.

In any publications that result from this research, neither my name nor any information from which I might be identified will be published without my consent.

Submission date

Page 2 of 3

initials date

Athletic Coaching Education - Athletic Training • Sport Management

Sport and Exercise Psychology. Physical Education Teacher Education 
Training: Effects on Immunity and Mood - 67 -

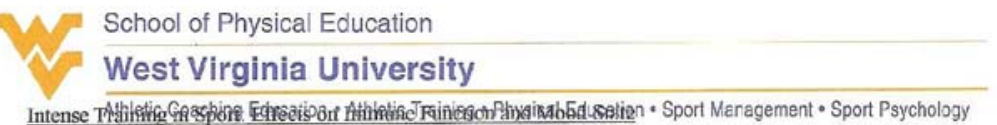

\section{Voluntary Participation}

Participation in this study is voluntary. I am free to withdraw my consent to participate in this study at any time. I will not receive financial considerations for participation.

Refusal to participate or withdrawal will not affect my future care, or my athletic status at West Virginia University or my class standing or grades, and will involve no penalty to me.

In the event new information becomes available that may affect my willingness to participate in this study, this information will be given to me so that I can make an informed decision about whether or not to continue participation.

I have been given the opportunity to ask questions about the research, and have received answers concerning areas I did not understand.

Upon signing this form, I will receive a copy

I willingly consent to participate in this research.

Signature of Subject or Subject's Legal Representative

Printed Name

Date Time

The participant has had the opportunity to have questions addressed. The participant willingly agrees to be in the study.

Signature of Investigator or Co-Investigator

Printed Name

Date Time

Contact Persons

In the event you experience any side effects or injury related to this research, you should contact Michelle Bartlettat (304) 685-5683. For more information about this research and about research-related risks or injury, you can contact Michelle Bartlett or Dr. Ed Etzel at (304) 293 7062. For information regarding your rights as a research subject, you may contact the Office of Research Compliance at (304)293-7073.

Submission date Page 3 of 3 
Training: Effects on Immunity and Mood - 68 -

School of Physical Education

West Virginia University

Athletic Coaching Education - Athletic Training - Physical Education - Sport Management - Sport Psychology

October 10,2005

Dear Madam:

My name is Michclle Bartlett. I am Doctoral Student focusing on Sport and Exercise Psychology within the School of Physical Education at West Virginia University. While enrolled in this program, students obtain both a master's and a doctoral degree. As part of my Master's Thesis, I am exploring the effects that intense training may have on the immune system and mood state.

As a Division 1 collegiate swimmer, we are asking that you participate in this study, which is described in more depth within the Consent Form that is attached. By signing this consent form, you are agreeing to participate in this study. Participation in this study will mean that you will complete survey forms that are used to determine mood state and life stressors. Additionally, you will provide small saliva samples to assess your general immune function. This information will be gathered solely for the purpose of this research, and will be kept completely confidential. Your information will be coded such that your name and information do not appear together. Your consenting to participate is completely voluntary, and you may withdraw at any time without penalty.

Should you have any questions about this program after reading this letter and the attached consent form, please feel free to contact Michelle Bartlett at 304-685-5683, or mbartle5@mix.wvu.edu.

Sincerely,

Michelle Bartlett

Doctoral Student

West Virginia University, Sport and Exercise Psychology
VIEST VIRGINIA UNIVERSITY Protection of Review Bosid for the

OCT 132005

$\times$. APPRONED

ExPIAES $\frac{10 \cdot 5 \cdot 06}{16694}$

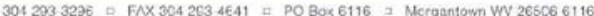


Appendix F

\section{Demographic Form}

Directions: Please check the corresponding blank.

1. What is your age group: _ـ 17-19 y/o _ 20-22 y/o _ $23+\mathrm{y} / \mathrm{o}$

2. What is your class standing: __ Freshman __ Sophomore __ Junior Senior __ Graduate

3. What is your ethnicity (optional): Caucasian African-American _other Hispanic Asian-American

4. What is your highest competitive level: __collegiate elite/Olympic

5. Please state the number of years you have been swimming competitively:

6. Please state the number of years you have been swimming on the West Virginia University Women’s Swimming team:

7. Please estimate the average number of upper respiratory tract infections you have per year (defined as symptoms of fever, pharyngitis, tonsillitis, rhinitis and swollen glands consistent with either overt bacterial infection or viral illness): 
Training: Effects on Immunity and Mood - 70 -

\section{Appendix G}

Review of Literature

Coaches and athletes are continuously trying to find ways to sustain improvement. There is a general consensus that training and performance have a doseresponse relationship, in which an increase in training volume and intensity leads to an increase in performance (Pyne et al., 2000). However, when increases in training are not coupled with adequate recovery, decrements in an athlete’s psychological and physical well being can result.

Currently, there is a need to establish solid methods for the early identification of maladaptions to intense training for athletes in order to make training adjustments before permanent effects on an athlete's health or athletic participation occurs. Recently, there has been much attention paid to the psychoneuroimmunological connection between factors indicative of overtraining. However, this is relatively new and much further study is needed to make unequivocal connections. This chapter is a review of the literature pertaining to: (1) the importance of studying negative training effects, (2) proposed markers of overtraining, (3) studies of immunological markers, (4) studies of psychological markers, and (4) the psychoneuroimmunological connection in overtraining. This chapter will conclude with a summary.

The Importance of Studying Negative Training Effects

The number of athletes affected and the plethora of detrimental effects that may impact an individual's athletic participation illustrates the importance of studying training and overtraining. The importance of optimal training is evident in the very small difference between winning and losing at elite levels. A competitively meaningful 
performance among athletes may only be on the order of 1\% (Lehmann et al., 1993). In a study of speed skaters during the 1988 Winter Olympics, the difference in mean velocity between all gold and silver medallists was .3\%. The mean difference between gold medallists and fourth place was only 1.4\%. A 3-6\% decrement in performance can mean the difference between first and last place at elite-level competition (Kuipers, 1998). In the $100 \mathrm{~m}$ sprint, a performance decrement of $1 \%$ can mean the difference between first and last place (MacKinnon, 2000). In swimming, a 4-5\% difference lies between Olympic qualifying times and world records (MacKinnon, 2000).

Concerning fatigue as a major symptom of overtraining, prolonged periods can interfere with preparation for major competitions. Mercurial performance at critical times in an athlete's career may lead to not making selective teams, loss of sponsorships and income, and possible premature retirement from sport altogether (MacKinnon, 2000). Studies were conducted surveying members of the 1996 Atlanta and 1998 Nagano Olympics teams on the major factors that they perceived as positive and negative influences during the games. The results illustrated the presence of overtraining as a negative influence in Olympic athletes. Analyses of the completed questionnaires indicated, out of 296 athletes from 30 different sports competing in the Atlanta games, 84 (28\%) of US Olympic athletes disclosed that they were over trained and that this had a negative effect on their performance. Thirty-five of these athletes named overtraining and not enough recovery as the main cause of negative Olympic performances (Gould \& Dieffenbach, 2002). From Nagano survey results 8 of 83, or $10 \%$ of US Olympic athletes representing 13 sports reported that they were overtrained and that this had a negative effect on their Olympic performance (Gould \& Dieffenbach, 
2002). Budgett et al. (2000) stated overtraining as a “common problem,” with prevalence in ten to twenty percent of elite endurance squads.

Budgett (1998) stated that overtraining mainly occurs in endurance athletes. MacKinnon (2000) explained that prevalence varies by sport and is deemed to be highest in endurance sports requiring high volume intense training such as swimming, triathlon, road cycling, rowing and running. Several studies do focus solely on overtraining in endurance sports, as well (i.e., Gabriel et al., 1998; Lehmann et al., 1993; Petibois et al., 2003; Robson, 2003). However, overtraining has been identified in most sports - both endurance and power sports. For example, it has been identified in basketball players, boxers, cyclists, gymnasts, rowers, swimmers, track and distance athletes and wrestlers (Hooper, MacKinnon, Howard, Gordon, \& Bachmann, 1995). The Atlanta survey study cited overtrained athletes in baseball, canoe and kayak, cycling, diving, fencing, field hockey, gymnastics, rowing, shooting, soccer, swimming, synchronized swimming, handball, volleyball, wrestling and yachting (Gould \& Dieffenbach, 2002). Other examples of prevalence include $60 \%$ of distance runners, $21 \%$ of Australian swimmers, and more than $50 \%$ of soccer players as overtrained (Smith, 2000). In evaluating distance running, Gould and Dieffenbach (2002) cited studies that found that $64 \%$ of males and $60 \%$ of females will report at least one instance of staleness throughout their running careers. 33\% of non-elite runners were found to experience at least one episode of staleness during their running careers.

Although often evident in elite-level sport, athletes at all performance levels are at risk of overtraining (Fry et al., 1991; Gould \& Dieffenbach, 2002). In studies of collegiate swimmers and other endurance athletes aggressively training, the reported 
yearly incidence of staleness equated roughly 10\%, ranging from a low of $7 \%$ to a high of $21 \%$ in various studies (Raglin \& Wilson, 2000). Concerning youth sport, Raglin and Wilson (2000) cited a study in which 31\% of adolescent distance runners reported staleness with episodes lastly about 3 weeks on average. Raglin and colleagues (2000) examined overtraining and staleness in youth swimmers across cultures. In this study, 231 youth swimmers, with an average age of 14.8 years, completed surveys that appraised training volumes, mood states and staleness. It was found that $35 \%$ of 231 young swimmers reported overtraining. The cross-cultural breakdown was 45\% Greek, 21\% Swedish, 34\% Japanese and 24\% American. Smith (2000) suggests that OTS is likely prevalent amid recreational athletes as well, but has not received the same attention in the field, for obvious reasons.

As long as athletes seek to improve their own or world record performances overtraining, both as a process and product, will endure. Taking into account the prevalence and impact of overtraining, it is of significance there has yet to be a single definitive test established that can show overtraining (Armstrong \& VanHeest, 2002; Fry et al., 1991; Hawley \& Schoene, 2003; Urhausen \& Kindermann, 2002) and the underlying mechanism for performance decrements is not fully known (Armstrong \& VanHeest, 2002; Urhausen \& Kinedermann, 2002). The onset and progression of overtraining syndrome is not easily predictable, and means for monitoring are more practicable in the research laboratory than in the athletic arena (Lehmann et al., 1993). Currently, there is a strong demand for relevant tools for the early diagnosis of overtraining (Urhausen \& Kindermann, 2002). 
Knowledge of overtraining and symptoms primarily stems from experience, single case observations, and cross- sectional and longitudinal studies during training. There is little data from prospective, controlled studies due to ethical convictions and inability to motivate individuals to train at such levels without foreseeable competitive goals (Lehmann et al., 1993). Overtraining has been difficult to study due to the lack of adequate experimental models of the condition (Foster, 1998). MacKinnon (2000) names two general models, albeit with disadvantages, used to study overtraining in athletes. In one model, physiological and psychological responses of the athlete are compared over a period of three to eight months in their natural environment. Measurements are compared between periods of high and low intensity or between athletes considered to be overtraining and well-trained. A disadvantage of this model is the inability to control for confounding variables, such as injury, diet, and social stressors. In the other model an attempt at overreaching is made by intensifying training over a period of four weeks (due to ethical reasons) with measures taken pre and post. However, there is much variability in the responses of athletes to intensified training. Also, considering that it is unlikely that four weeks of intense training will induce overtraining in an athlete, most understandings from studies using this model are extrapolations from experimental studies of overreaching (Foster, 1998; Lehmann et al., 1993).

Individual differences in response to various training loads have been cited as playing a major role in the lack of a clear and consistent marker of overtraining (e.g., Budgett, 1998; Hassmen, 1998; Lehmann et al., 1993; MacKinnon, 2000; Smith, 2000) Athletes' demonstrate diversified combinations of symptoms, with varying degrees of 
severity and no specific pattern relating to athletic event. Clinical presentation is best ascertained with a combination of markers and symptoms (Mackinnon, 2000; Smith, 2000; 2003). Fry et al, (1991) and Hartmann and Mester (2000) suggested that the baseline measurements must be taken for individual athletes in a normally trained state as their own standard. Results are not applicable in relating to population norms because changes indicative of the onset overtraining may be too sensitive to be noted. Athletes may be in the stages of grossly overtraining before changes are heeded clinically significant in terms of deviating from the normal population. Also, inter-individual differences in recovery potential, exercise capacity, non-training stress, and stress tolerance contribute to differences in vulnerability by athletes under identical training conditions (Kellman, 2003). Fry et al.(1991) called for a subjective battery of tests for training athletes covering several parameters associated with unbalanced homeostasis taken as a baseline measure. Evaluating athletes individually, monitoring them regularly and comparing data longitudinally could be a key in monitoring overtraining (Kellman, 2003).

In order to progress in advances toward prevention and early recognition of the overtraining, Hassmen (1998) called for increased understanding and advances in several areas. These include: the lack of accepted tests and standard criteria to unerringly place athletes on the overtraining-response continuum, lack of a reliable and easily managed method to monitor responses to training, the need to define markers of recovery, and the integration of these. 
Training: Effects on Immunity and Mood - 76 -

\section{Proposed Markers of Overtraining}

There have been many proposed markers in the early identification of overtraining. However, most markers have been equivocal in the literature. There is argument over whether noted changes reflect the natural stress of training, rather than a breakdown in adaptation mechanisms. Concerning the value of discovering markers of overtraining, it has been discussed amongst researchers as to whether they are capable of standing as early warning signs, or merely being confirmatory in nature (Hooper et al., 1995; Kentta \& Hassmen, 2002). A decrease in markers maximum performance, such as VO2 max (peak oxygen consumption), tends to be reduced in overtrained athletes (Lehmann et al., 1992; Stone et al., 1991) but unchanged values are not unusual (Urhausen, Gabriel, Weiler, \& Kindermann, 1998; Urhausen \& Kindermann, 2002).

An increase in resting heart rate has been reported (Budgett, 1991) as well as no change (Hooper et al., 1995). Maximum heart rate has been shown to be slightly, but significantly, reduced in overtrained athletes (Gleeson, 1998; Lehmann et al., 1992; Urhausen, Gabriel, \& Weiler, 1998; Varlet-Marie, Maso, Lac, \& Brun, 2004) However, the average decrease is approximately 3 to 5 beats/min, which impairs the usefulness as a marker in practice (Urhausen \& Kindermann, 2002).

Maximum lactate levels have been tested as markers of overtraining. Lactic acid is the end product of fast anaerobic glycolysis, which is then buffered in the muscle and blood to form the salt, lactate (Stone et al., 1991) Lowered levels are representative of a lowered threshold for anaerobic muscle contraction (Urhausen \& Kindermann, 2002). Maximum levels have been shown to decrease in overtrained athletes (Budgett, 1991; Lehmann et al., 1993; Stone et al., 1991; Urhausen et al., 1998; Varlet-Marie et al., 
2004). Hooper et al. (1995), and Lehmann et al. (1992) have showed no change in maximum lactate levels in overtrained athletes. Urhausen and Kindermann (2002) further discredit maximum lactate levels as a marker by stating that changes in the levels do not diagnose overtraining, but merely exclude other training errors.

Measures of performance as early markers of overtraining prove insufficient, for these markers usually just confirm already evident decreases in performance. The determination of hormone concentrations for monitoring overtraining and potential overtraining have been a focus of study in sport science over the past 15 years (Smith \& Norris, 2000). It has been speculated that a hormonal mediated central dysregulation occurs during the pathogenesis of the overtraining syndrome. The hypothalamopituitary-adrenal axis has been indicated in playing an essential role (Barron, Noakes, Levy, Smith, \& Millar, 1985; Urhausen et al., 1998). Several extensive reviews and investigations have examined the endocrinological changes that may accompany overtraining (Armstrong \& VanHeest, 2002; Barron et al., 1985; Stone et al., 1991; Urhausen et al., 1998). Hormones that have been examined as markers of overtraining include catecholamines, epinephrine, norepinephrine, cortisol and testosterone, among others. Literature is also equivocal on the value of hormones as markers.

\section{Studies of Immunological Markers}

There have been suggestions that altered immune reactions might provide an early warning sign that an athlete is overtraining (McGrew, 1995). A common symptom of overtraining is immunosupression, characterized by slower wound healing (Newsholme, 1994) and an increased susceptibility to infection, namely upper respiratory tract infections (URTI) (Fitzgerald, 1991; MacKinnon, 2000; McKenzie, 
1999; Neiman, 1994; Shepard \& Shek, 1998; Smith \& Norris, 2002). For example, in a study of 24 competitive swimmers by MacKinnon \& Hooper (1996), training was intensified over 4 weeks resulting in 33\% of the swimmers showing symptoms of shortterm overtraining. Of the 24 swimmers, $42 \%$ were diagnosed with URTI’s. A study of 25 elite level speed skaters by Foster (1998) attempted to define the percentages of illness that could be correlated to training load. In analyzing training journals recording a range of six months to three years, it was found that $84 \%$ of illnesses could be explained by a preceding spike in training load above the individual training threshold. In a study of elite runners by Verde et al. (1992), respiratory illness developed in 3 of 10 runners when a 38\% increase of training was induced over 3 weeks. Newsholme (1994) suggested that URTIs are more common in athletes during intense periods of training than in the general population. In a study on over 500 runners, the yearly incidence of URTI was shown to be related to training volume. URTI incidence was two times higher in runners averaging 15-27 km per week and 3.5 times higher in those averaging more than $27 \mathrm{~km}$ per week than for those training less than $15 \mathrm{~km}$ per week (Heath et al, 1991).

Within the immune system, mechanisms against invading viral infections include physical and mechanical barriers, such as nasal filtration, mucus secretion and expectoration, and the endothelial membrane. Biological barriers include NK (natural killer) cells and cytotoxic lymphocytes in the lysis infected cells, interferon in the enhancement NK cell activity and inhibition of viral replication, specific antibodies, such as IgA, which prevent membrance penetration by the virus, phagocytes, which ingest viral particles, and soluble elements such as complement and acute phase 
proteins, which attract the immune response to effected regions (Shephard \& Shek, 2001). Host defense at mucosal surfaces from invading pathogens is mostly mediated by the family of secretory immunoglobins (Ig) (MacKinnon, 1996). The principal antibody, or immunoglobin, found in external secretions, such as mucous, tears, and saliva, is Immunoglobin A, or IgA (Gleeson, 2002, Nieman, 1994). The most convenient secretory fluid for the assessment of IgA is saliva (sIgA). The noninvasiveness of saliva sampling and ease of sampling makes assessment of sIgA particularly useful for repeated measures and non-laboratory settings (Evan et al., 2000; Gleeson, Hall, et al., 1999).

It has been argued that levels of total secretory IgA measured in saliva are representative of immunological activity in the immune system as a whole (Evans et al., 2000; McDowell et al., 1992). Other researchers suggest that measurement of total sIgA may not directly reflect the ability to ward off specific respiratory infections, but that it does have a role in limiting respiratory infections (Gleeson, Hall, et al., 1999). Levels of sIgA have been shown to highly correlate with resistance to the viruses that cause URTI’s (MacKinnon, 2000; McKenzie, 1999). Decreases in mucosal IgA could have an important influence on susceptibility to URTI's in the first few hours following viral exposure, considering that the function of sIgA is to inhibit the viral particles from attaching to the respiratory lining (Shephard \& Shek, 2001). To date, salivary IgA concentration is one of the only immune parameter to be directly associated with the appearance of URTI’s (MacKinnon, 2000) and used a marker for athletes at risk of developing an URTI (Gleeson, 2002; Shephard \& Shek, 1998). In a study by Gleeson, Hall, et al. (1999) there was a statistically significant correlation between the number of 
respiratory infections throughout the season and baseline salivary IgA levels. Low levels of salivary IgA have been documented in overtrained athletes and decreases in salivary IgA concentrations can be observed during intensified training in elite swimmers (MacKinnon, 1996). For example, in a study by MacKinnon and Hooper (1994) a significantly lower concentration of salivary IgA was reported for a group of elite swimmers throughout a six-month season who were clinically diagnosed as suffering from overtraining, compared to teammates that were considered well-trained.

Athletes, as well as the general population, are quite able to correctly diagnose symptoms of upper respiratory tract infections (URTI's) such as runny nose, sore throat, and cough in combination with fatigue, headache and fever (Cohen et al., 1991). Salivary IgA levels show an inverse correlation with the number of URTI's experienced (MacKinnon, 2000). Therefore, the presence of an URTI may be a variable reflected in lowered sIgA levels. Also, in some athletes a viral infection may lead to weakened state known as "post viral fatigue syndrome” (Nieman, 2000). The residual effects of an URTI may remain evident in lowered sIgA levels, even after symptoms of the illness have subsided.

Daily intense exercise may have a cumulative suppressant effect on sIgA output. As exemplified in the data of MacKinnon and Hooper (1994) and Tharp and Barnes (1990) where as training load accumulated over the course of a competitive season, sIgA levels decreased. In another study, Gleeson et al.(1995) found a significant decrease in both pre- and post-exercise sIgA levels over a seven-month training period with elite swimmers compared to age-matched non-athletes. Pre-exercise IgA levels were $4.1 \%$ lower for each additional month of training and 5.8\% lower for each 
additional infection. The data from this study also indicated that the elite swimmers with low sIgA levels at the beginning of the season were associated with a higher risk of RTIs during the season (Gleeson, Hall, et al., 1999).

Salivary IgA has several advantages as a measure in the early indication of overtraining. Studies utilizing sIgA assessments show good repeat measure reliability. Sampling is non-invasive and can be taken with little trouble or discomfort to the subject (Evans et al., 2000). The depression of salivary IgA levels seems to be one of the more consistent indicators of overtraining (Shephard \& Shek, 1998) and have been cited as the most promising immune system indicator of overtraining by researchers (MacKinnon, 2000; McKenzie, 1999; Shepard \& Shek, 1998). Psychological stress also can impair aspects of immune function and increase susceptibility URTI's and effect sIgA levels (Shephard \& Shek, 2001).

Studies of Psychological Markers

Some researches have argued that psychological testing is most effective in detecting overtraining at an early stage (Kentta \& Hassmen, 2002; O’Connor, 1998; Shephard \& Shek, 1994). Athletes that are overtrained tend to show a trend in the order of the indices affected. The first noticeable change is suggested to be within psychological factors, followed by medical condition, and lastly performance (McKenzie, 1999) Psychological and emotional symptoms indicative of overtraining include unstable and/or depressed moods, lowered tolerance of frustration and stress, feelings of helplessness and worthlessness, a fear of competition, anxiety and apathy (Fry et al., 1991; Henschen, 2000). Armstrong \& VanHeest (2002) suggest that the 
symptoms seen in the overtrained athlete are remarkably similar to symptoms of clinical depression.

Shephard and Shek (1994), as well as Davis and Colbert (1997), concluded via a review of the research on markers of overtraining that psychological assessments are more consistent across athletes. This consistency coupled with the inconsistency of physiological markers, as well as the early onset of psychological symptoms, suggests psychological testing as a more effective way to assess overtraining in athletes.

A majority of the research on psychological correlates of overtraining has used the Profile of Mood States- the POMS (Kentta \& Hassmen, 2002; Morgan et al., 1987; O’Connor, 1997; Raglin \& Wilson, 2000; Veale, 1991). The POMS (McNair et al., 1971) is a 65-item self-report questionnaire, which yields a global measure of mood as well as the stable mood states of tension, anger, vigor, fatigue, and confusion (Morgan et al.,1987; Raglin \& Wilson, 2000; Veale, 1991). The total measure of mood state is calculated by adding the five negative mood states and subtracting the positive factor of vigor. A constant of 100 is added to this value to prevent negative scores (Morgan et al., 1987; Raglin \& Wilson, 2000). The POMS has four standard instructional response sets, however, when used in overtraining studies, instructions to respond according to how one has been feeling “the last week including today” (Morgan, et al.,1987; Raglin \& Wilson, 2000). Use of the POMS has evinced a consistent response to heavy training and early overtraining by athletes, most notable are a decrease in vigor and an increase in fatigue (Morgan et al., 1987; Raglin et al., 1991; Raglin \& Wilson, 2000; Veale, 1991; Verde et al., 1992). In a study on potential markers of heavy training in elite distance runners, Verde et al. (1992) found that mood state assessment via the POMS 
was more sensitive at measuring early stages of overtraining than physiological measures. The only of ten measures in which a consistent response was displayed was with the POMS. Further evidence of consistency concerns gender differences with using the POMS. Raglin, Morgan, \& O’Connor (1987) conducted a study of NCAA varsity male and female swimmers to assess psychological responses to altered training levels. Both the male and female swimmers displayed similar mood state responses.

Evidence of the POMS being a highly sensitive early warning marker of overtraining has been demonstrated in several studies on competitive swimmers by Morgan et al. (1987; 1988). In summary, they have found that there is a dose-response relationship in which in increases in training are associated with increased mood disturbance, the earliest psychological changes measured by the POMS are an increase in fatigue and a decrease in vigor. In a study on 14 female college swimmers, POMS scores indicated that mood states worsened as training volume increased interpretation of mood profiles may successful predict the physiological and performance changes associated with overtraining (O’Connor, Morgan, Raglin, Barksdale, \& Kalin, 1989).

A disadvantage to using the POMS is that the intent of the questions is fairly obvious to the respondent (Verde et al.,1992). Respondents, fearful that they will be mandated to reduce or possible stop training, may falsify answers. Also, the POMS does not provide information about the cause of the mood change, only that there has been (Kellman, 2002).

\section{Psychoneuroimmunological Connection}

The promise of the psychological testing is disputed by the possibility that these mood changes may reflect underlying biochemical or immunological changes that are 
communicated to the brain via hormones and cytokines (Gleeson, 2002). O’Connor (1998) noted that variations of mood often correlate with those of physiological markers.

A premise of psychoneuroimmunology, or the study of behavior-brain-immune interrelationships (Armstrong \& VanHeest, 2002), is how the immune system is influenced by our emotional states, our feeling states: chronic states of stress- such as overtraining (Evan et al., 2000). The immune system is extremely sensitive to stress both physiological and psychological (Gleeson, 1998). Training and competing at elite levels can involve psychological and physical stress. Several researchers have stated that the effects of both stresses on the immune system are additive (Clow \& Hucklebridge, 2001; Fitzgerald, 1991; MacKinnon \& Hooper, 1994; MacKinnon, 1997; Pyne \& Gleeson, 1998). Therefore, athletes are especially vulnerable. Chronic life stress, unrelated to sport, can also negatively affect immune status (Perna, Schneiderman, \& LaPerriere, 1997). For instance, Cohen, Tyrell, and Smith (1991) found a dose-response relationship between self-reported life stress and immunosupression when participants were exposed to a rhinovirus. Endurance training causes effects similar to those seen during chronic psychological stress (Clow \& Hucklebridge, 2001), which explains the need to assess life stressors of the participants. Studies comparing mood state and sIgA levels are equivocal. In a study of male collegiate swimmers, no significant correlations were shown between the global POMS score and IgA levels. No significant correlations were found between mood state and IgA levels assessed at morning and evening, before and after exercise in a study by Dimitriou, Sharp \& Doherty (2002). However, in elite swimmers monitored over 7 
months, psychological stress was significantly higher in periods of infection-prone periods, characterized by sIgA levels less than $30 \mathrm{mg} / \mathrm{L}$, than infection-free periods (Gleeson, McDonald, et al., 1999). The data of MacKinnon and Hooper (1994) on both elite swimmers and runners show a significant correlation between sIgA concentrations and indicators of mood state disturbance, as measured by the POMS.

Summary

Numerous studies exemplify the relationships of intense training on immunological and/or psychological parameters (i.e., Gabriel et al., 1998; Gleeson, Hall, et al., 1999; Gleeson, McDonald, et al., 1999; Gleeson et al., 1995; MacKinnon \& Hooper, 1996; McDowell et al., 1992; Morgan et al., 1987; Morgan et al., 1988; Raglin et al., 1991; Tharp \& Barnes, 1990; Verde et al., 1992)

MacKinnon and Hooper (1994) suggested that mucosal immunity may be compromised by daily exercise of at least moderate intensity as undertaken by competitive athletes. Thus, an athlete may show impaired immunity as a result of training but may not be actually overtraining. However, the product of impaired immunity and a disturbed Profile of Mood States could be a significant marker of negative overtraining effects. By incorporating the use of the POMS questionnairerecognized as the most promising method of psychological testing in marking overtraining (Morgan et al.,1987; Raglin, et al., 1991; Raglin \& Wilson, 2000; Veale, 1991; Verde et al., 1992), and levels of sIgA- recognized as the most promising physiological measure in marking overtraining (McKenzie, 1999; MacKinnon, 2000; Shepard \& Shek, 1998), it may be that over the course of the competitive season, if an athlete demonstrates changes in sIgA levels over the season, the POMS will also reflect 
those differences and vice versa. This is under the premise of psychoneuroimmunology - the interdisciplinary study of the behavioral- neural- endocrine- immune system interactions (Ader \& Cohen, 1995), or the relationship between psychological variables, the nervous system, and the immune system (LaPerriere et al, 1994; Mcgrew, 1995).

Being that the activities of the immune system in the psychological factor of mood state will be reflected in each other. 


\section{References}

Ader, R., \& Cohen, N. (1995). Psychoneuroimmunology: Interactions between the nervous system and the immune system [Electronic version]. Lancet, 345 (8942), 99-106.

Armstrong, E., \& VanHeest, J.L. (2002). The unknown mechanism of the overtraining Syndrome: Clues from depression and psychoneuroimmunology. Sports Medicine, 32, 185-209.

Barron, J., Noakes, T., Levy, W., Smith, C., \& Millar, R. (1985). Hypothalamic dysfunction in overtrained athletes. Journal of Clinical Endocrinology and Metabolism, 60, 803-806.

Budgett, R. (1991). Overtraining syndrome. British Journal of Sports Medicine, 24, 231-236.

Budgett, R. (1998). Fatigue and underperformance in athletes: The overtraining syndrome.

British Journal of Sports Medicine, 32, 107-110.

Budgett, R., Newsholme, E., Lehmann, M., Sharp, C., Jones, D., \& Peto, T., et al. (2000). Redefining the overtraining syndrome as the unexplained underperformance syndrome. Sports Medicine, 34, 67-68.

Clow, A. \& Hucklebridge, F. (2001) The impact of psychological stress on immune function in the athletic population. Exercise Immunology Review, 7, 5-17.

Cohen, S., Tyrell, D.A., and Smith, A.P. (1991). Psychological stress and susceptibility to the common cold. New England Journal of Medicine, 325, 606-612.

Davis, M. \& Colbert, L.H. (1997). The athlete’s immune system, intense exercise, and 
Overtraining. In D.R Lamb \& R. Murray (Eds.), Perspectives in exercise science and sports medicine: Vol. 10. Optimizing sport performance. Carmel, IN:

Cooper.

Dean, J., Whelan, J.P., \& Meyers, A.W. (1990, September) An incredibly quick way to assess mood states: The incredibly short POMS. Paper presented at the Association for the Advancement of Applied Sport Psychology, San Antonio, TX.

Dimitriou, L., Sharp, N.C., \& Doherty, M. (2002). Circadian effects in the acute responses of salivary cortisol and IgA in well-trained swimmers. British Journal of Sports Medicine, 36, 260-264.

Evans, P., Hucklebridge, F., \& Clow, A. (2000). Mind, Immunity, and Health: The science of psychoneuroimmunology. Free Association Books: London/ NY.

Fitzgerald, L. (1991). Overtraining increases the susceptibility to infection. International Journal of Sports Medicine, 12, S5-S8.

Foster, C. (1998). Monitoring training in athletes with reference to overtraining syndrome. Medicine and Science in Sports and Exercise, 30, 1164-1168.

Fry, R.W., Morton, A.R., \& Keast, D. (1991). Overtraining in athletes: An update. Sports Medicine, 12, 32-65.

Gabriel, H.W., Urhausen, A., Valet, G., Heidelbach, U., \& Kindermann, W. (1998). Overtraining and immune system: a prospective longitudinal study in endurance athletes. Medicine and Science in Sport and Exercise, 30, 11511157.

Gleeson, M. (1998). Overtraining and stress responses. Sports Exercise and Injury, 4, 
62-68.

Gleeson, M. (2002). Biochemical and immunological markers of overtraining. Journal Of Sports Science and Medicine, 1, 31-41.

Gleeson, M., Hall, S, McDonald, W., Flanagan, A., \& Clancy (1999). Salivary IgA subclasses and infection risk in elite swimmers. Immunology and Cell Biology, 77, 351-355.

Gleeson, M., McDonald, W.A., Cripps, A.W., Pyne, D.B., Clancy, R.L., \& Fricker, P.A. (1995). The effect on immunity of long term intensive training on elite swimmers. Clinical Experimental Immunology, 102, 210-216.

Gleeson, M., McDonald, W.A., Pyne, D.B, Cripps, A.W., Francis, J.L., \& Fricker, P.A., et al. (1999). Salivary IgA levels and infection risk in elite swimmers. Medicine and Science in Sports and Exercise, 31, 67-73.

Gould, D. \& Dieffenbach, K. (2002). Overtraining , under recovery, and burnout in sport. In M. Kellman (Ed.), Enhancing recovery: Preventing underperformance in athletes (pp. 25-35). Champaign, IL: Human Kinetics.

Hartman, U., \& Meister, J. (2000). Training and overtraining markers in selected sport events. Medicine and Science in Sport and Exercise, 32, 209-215.

Hassmen, P. (1998). Overtraining and recovery: A conceptual model. Sports Medicine, 26, 1622-1542.

Hawley, C., \& Schoene, J. (2003). Overtraining syndrome. Physician \& Sportsmedicine. 31 (6), 25-32.

Heath, G.W., Ford, E.S., Craven. T.E., Macera, C.A., Jackson, K.L., \& Pate, R.E. (1991). Exercise and the incidence of upper respiratory tract infections. 
Medicine and Science in Sport and Exercise, 23, 152-157.

Henschen, K. (2000). Maladaptive fatigue syndrome and emotions in sport. In Y.L. Hanin (Eds.), Emotions in sport (pp. 191-207). Champaign, IL: Human Kinetics.

Hooper, S., MacKinnon, L.T.,Gordon, R.D., \& Bachmann, A.W. (1993). Hormonal responses of elite swimmers to overtraining. Medicine and Science in Sport and Exercise, 25, 741-747.

Hooper, S., MacKinnon, L.T., Howard, A., Gordon, R.D., \& Bachmann, A.W. (1995). Markers for monitoring overtraining and recovery. Medicine and Science in Sport and Exercise, 27 (1), 106-112.

Kellman, M. (2002). Psychological assessment of under recovery. In M. Kellman (Ed.) Enhancing recovery: Preventing underperformance in Athletes (pp. 3-24). Champaign, IL: Human Kinetics.

Kellman, M. (2003). Under recovery and overtraining: Different concepts- similar impact? Olympic Coach, 15 (3), 4-7.

Kentta, G., \& Hassmen, P.(2002). Under recovery and overtraining: A conceptual model. In M. Kellman (Ed.) Enhancing recovery: Preventing underperformance in athletes (pp. 3-24). Champaign, IL: Human Kinetics.

Kuipers, H. (1998). Training and overtraining: An introduction. Medicine and Science in Sport and Exercise, 30 (7), 1137-1139.

LaPerriere, A., Iron son, G., Antonio, M.H., Schneiderman, N., Klimas, N., \& Fletcher, M.A. (1994) Exercise and psychoneuroimmunology. Medicine and Science in Sport and Exercise, 26 (2), 182-190.

Lehmann, M., Foster, C., \& Keul, J. (1993). Overtraining in endurance athletes: a brief 
review. Medicine and Science in Sports and Exercise, 25 (7), 854-862.

Lehmann, M., Foster, C., Dickhuth, H., \& Gastmann, U. (1998). Autonomic imbalance hypothesis and overtraining syndrome. Medicine and Science in Sport and Exercise, 30 (7), 1140-1145.

Lehmann, M, Gastmann, U., Petersen, K.G., Bachl, N., Seidel, A., \& Khalaf, A.N., et al. (1992) Training-overtraining: Performance and hormone levels, after a defined increase in training volume versus intensity in experienced middle and long distance runners. British Journal of Sports Medicine, 26, 233-242.

MacKinnon, L.T. (1996). Immunoglobin, antibody, and exercise. Exercise Immunology Review, 2, 1-35.

MacKinnon, L.T. (1997). Immunity in athletes. International Journal of Sports Medicine, 18, S62-S62.

MacKinnon, L.T. (2000). Overtraining effects on immunity and performance in athletes. Immunology and Cell Biology, 78, 502-509.

MacKinnon, L.T., \& Hooper, S. (1994). Mucosal (secretory) immune system responses to exercise of varying intensity and during overtraining. International Journal of Sports Medicine, 15, S179-S183.

MacKinnon, L.T., \& Hooper, S.L. (1996). Plasma glutamine and upper respiratory tract infections during intensified training in swimmers. Medicine and Science in Sport and Exercise, 28, 285-290.

Maier, S.F., \& Watkins, L.R. (1998). Cytokines for psychologists: Implications of bidirectional immune-to-brain communication for understanding behavior, mood, and cognition. Psychological Review, 105 (1), 83-107. 
Maier, S.F., Watkins, L.R., \& Fleshner, M. (1994). Psychoneuroimmunology: The interface between brain, behavior, and immunity. American Psychologist, 49 (12),1004-1017.

McDowell, S.L., Hughes, R.A., Hughes, R.J., Housh, D.J., Housh, T.J., \& Johnson, G.O. (1992). The effect of exhaustive exercise on salivary Immunoglobin A. The Journal of Sports Medicine and Physical Fitness, 32, 412-415.

McGrew, C.A. (1995) Exercise and Immunology. Sports Medicine and Arthroscopy Review, 3, 101-106.

McKenzie, D.C. (1999). Markers of excessive exercise. Canadian Journal of Applied Physiology, 24 (1), 66-73.

McNair, D., Lorr, M., \& Droppelman, L. (1971). Profile of Mood State manual. San Diego: Educational and Industrial Testing Service.

Morgan, W.P., Brown, D.R., Raglin, J.S., O’Connor, P.J., \& Ellickson, K.A.(1987). Psychological monitoring of overtraining and staleness. British Journal of Sports Medicine. 21 (3), 107-114.

Morgan, W.P., Costill, D.L., Flynn, M.G., Raglin, J.S., \& O’Connor, J.P. (1988) Mood disturbance following increased training in swimmers. Medicine and Science in Sports and Exercise, 20 (4), 408-414.

Neiman, D.C. (1994). Exercise, infection and immunity. International Journal of Sports Medicine, 15, S131-S141.

Neiman, D.C. (2000) Is infection risk linked to exercise workload? Medicine and Science in Sports and Exercise, 32 (7), S406-S411.

Newsholme, E.A. (1994) Biochemical mechanisms to explain immunosupression in 
well- trained and overtrained athletes. International Journal of Sports Medicine, 15, S142-S147.

O’Connor, P.J. (1998). Overtraining and staleness. In W.P. Morgan (Ed.), Physical activity and mental health (pp.149-160). Washington, DC: Taylor \& Francis.

O’Connor, P.J., Morgan, W.P., Raglin, J.S., Barksdale, C.M., \& Kalin, N.H. (1989). Mood state and salivary cortisol levels following overtraining in female swimmers. Psychoneuroendocrinology, 14 (4), 303-310.

Perna, F.M., Schneiderman, N., \& LaPerriere, A. (1997). Psychological stress, exercise, and immunity. International Journal of Sports Medicine, 18, S78-S83.

Petibois, C., Cazorla, G., Poortmans, J., \& Deleris, G. (2003). Biochemical aspects of overtraining in endurance sports: The metabolism alteration process syndrome. Sports Medicine, 33 (2), 83-94.

Putlur, P., Foster, C., Miskowski, J.A., Kane, M., Burton, S.E., \& Scheett, T.P., et al. (2004). Alteration of immune function in women collegiate soccer players and college students. Journal of Sports Science and Medicine, 3, 234-243.

Pyne, D.B., \& Gleeson, M. (1998). Effects of intensive exercise training on immunity in athletes. International Journal of Sports Medicine, 19, S183-S194.

Pyne, D.B., Gleeson, M., McDonald, W.A., Clancy, R.L., Perry Jr., C., \& Fricker, P.A. (2000). Training strategies to maintain immunocompetence in athletes. International Journal of Sports Medicine, 21 (supp. 1), S51-S60.

Pyne, D.B., Gray, A.B., \& McDonald, W.A. (1995). Exercise and Immunity. In J. Bloomfield, P.A. Fricker \& K.D. Fitch (Eds.), Science and medicine in sport ( $\left.2^{\text {nd }} e d.\right)$. Cambridge, MA: Blackwell Science, 601-615. 
Raglin, J.S., Morgan, W.P., \& O’Connor, P.J. (1991). Changes in mood states during training in female and male college swimmers. International Journal of Sports Medicine. 12 (6), 585-589.

Raglin, J.S., \& Wilson, G. (2000). Overtraining in athletes. In Y.L. Hanin (Eds.), Emotions In Sport (pp. 191-207). Champaign, IL: Human Kinetics.

Robson, P.J. (2003). Elucidating the unexplained underperformance syndrome in endurance athletes: The Interleukin-6 hypothesis. Sports Medicine, 33 (10), 771781.

Salimetrics. LLC. (2005). Collection advice: Salivary samples from human subjects. [Brochure]. State College, PA: Author.

Shephard, R.J., \& Shek, P.N. (1994). Potential impact of physical activity and sport on the immune system: a brief review. British Journal of Sports Medicine, 28, 347355.

Shephard, R.J., Shek, P.N. (1998). Acute and chronic overexertion: Do depressed immune responses provide useful markers? International Journal of Sports Medicine, 19, 159-171.

Shephard, R.J., Shek, P.N. (2001) Physical activity and upper respiratory infection. In R. Ader, D. Felton, \& N.Cohen (Eds.), Psychoneuroimmunology, (Vol.2 ), pp. $511-521$.

Smith, L.L. (2000). Cytokine hypothesis of overtraining: a physiological adaptation to excessive stress? Medicine and Science in Sport and Exercise, 32, 317-331.

Smith, L.L. (2003). Overtraining, excessive exercise, and altered immunity: Is this a T Helper-1 versus T Helper-2 lymphocyte response? Sports Medicine, 33, 347- 
Training: Effects on Immunity and Mood - 95 -

364.

Smith, D.J., \& Norris, S.R. (2002). Training load and monitoring an athlete’s tolerance for endurance training. In M. Kellman (Ed.), Enhancing recovery: Preventing underperformance in athletes (pp. 3-24). Champaign, IL: Human Kinetics.

Stone, M.H., Keith, J.T., Kearney, S.J., Fleck, Wilson, G.D., \& Triplett, N.T. (1991). Overtraining: A review of the signs, symptoms, and possible causes. Journal of Applied Sports Sciences Research, 5 (1), 35-50.

Tharp, G.D. \& Barnes, M.W. (1990). Reduction of saliva immunoglobin levels by swim training. European Journal of Applied Physiology, 60, 61-64.

Urhausen, A., Gabriel, H., \& Kindermann, W. (1998). Impaired pituitary hormonal response to exhaustive exercise in overtrained endurance athletes. Medicine and Science in Sport and Exercise, 30, 407-414.

Urhausen, A., Gabriel, H., \& Weiler, B., \& Kindermann, W. (1998). Ergometric and psychological findings during overtraining: A long-term follow-up study in endurance athletes. International Journal of Sports Medicine, 19, 114-120.

Urhausen, A., \& Kindermann, W. (2002). Diagnosis of overtraining: What tools do we have? Sports Medicine, 32, 95-102.

Varlet- Marie, E., Maso, F., Lac, G., \& Brun, J. (2004). Hemorheological disturbances in the overtraining syndrome. Clinical Hemorheology and Microcirculation, 30, 211-218.

Veale, D.M.W. (1991). Psychological aspects of staleness and dependence on exercise. International Journal of Sports Medicine, 12 (S1), S19-S21. 
Training: Effects on Immunity and Mood - 96 -

Verde, T., Thomas, S., \& Shepard, R. (1992). Potential markers of heavy training in highly trained distance runners. British Journal of Sports Medicine, 26, 167-175. 